\title{
Equidimensional Adic Eigenvarieties for Groups With Discrete Series
}

\author{
Daniel Robert Gulotta
}

Submitted in partial fulfillment of the requirements for the degree of

Doctor of Philosophy

in the Graduate School of Arts and Sciences

COLUMBIA UNIVERSITY

2018 
(C) 2018

Daniel Robert Gulotta

All rights reserved 


\section{ABSTRACT \\ Equidimensional Adic Eigenvarieties for Groups with Discrete Series Daniel Robert Gulotta}

We extend Urban's construction of eigenvarieties for reductive groups $G$ such that $G(\mathbb{R})$ has discrete series to include characteristic $p$ points at the boundary of weight space. In order to perform this construction, we define a notion of "locally analytic" functions and distributions on a locally $\mathbb{Q}_{p}$-analytic manifold taking values in a complete Tate $\mathbb{Z}_{p}$-algebra in which $p$ is not necessarily invertible. Our definition agrees with the definition of locally analytic distributions on $p$-adic Lie groups given by Johansson and Newton. 


\section{Contents}

Acknowledgments $\quad$ iii

1. Introduction 1

2. Modules over complete Tate rings 4

2.1. Definitions 4

2.2. Spectral theory 5

$\begin{array}{lll}2.3 . & \text { Norms } & 6\end{array}$

\begin{tabular}{llll}
\hline 3. & Locally analytic functions and distributions & 7
\end{tabular}

$\begin{array}{lll}\text { 3.1. Preliminaries } & 7\end{array}$

3.2. Definitions 9

\begin{tabular}{lll}
\hline 3.3. & Properties of locally analytic functions and distributions & 12
\end{tabular}

$\begin{array}{ll}\text { 3.4. Gluing } & 17\end{array}$

3.5. Geometric interpretation of distributions 18

\begin{tabular}{lll}
\hline 4. & Overconvergent cohomology & 19
\end{tabular}

$\begin{array}{lll}4.1 . & \text { Locally symmetric spaces } & 19\end{array}$

4.2. Hecke action 21

4.3. Topological properties of Hecke operators 23

4.4. Characteristic power series 24

5. Eisenstein and cuspidal contributions to characteristic power series 25

5.1. Preliminaries 25

5.2. Cohomology of the Borel-Serre boundary 27

5.3. Hecke action 29

5.4. Image of the map $R \pi_{*} \iota^{*} \quad 30$

5.5. The complex $C_{G, K^{p}, \lambda, \text { cusp }}^{\bullet} 34$

\begin{tabular}{lll}
\hline 6. & Theory of determinants & 35
\end{tabular} 
7. Construction of the eigenvariety 38

7.1. Weight space and Fredholm series 38

$\begin{array}{lll}7.2 . & \text { Pieces of the eigenvariety } & 39\end{array}$

\begin{tabular}{lll}
\hline 7.3. & Gluing & 41
\end{tabular}

$\begin{array}{ll}\text { References } & 42\end{array}$ 


\section{ACKNOWLEDGMENTS}

I would like to thank my advisor, Eric Urban, for suggesting my thesis problem and for many helpful discussions. David Hansen and Shrenik Shah also patiently answered many of my questions. I benefited from interactions with many other Columbia faculty members, including Ali Altug, Johan de Jong, Dorian Goldfeld, Michael Harris, Daniel Litt, Chao Li, Wei Zhang, and Yihang Zhu.

My fellow graduate students taught me a lot and made my time at Columbia more enjoyable. I would like to thank Pak-Hin Lee for many adventures, and Rahul Krishna and Vivek Pal for guidance during my early years as a graduate student. I found the learning seminars at Columbia very helpful; I thank the above mentioned students as well as Karol Koziol, Shizhang Li, Qixiao Ma, Sam Mundy, and Remy van Dobben de Bruyn for their contributions to these seminars. I also enjoyed hanging out with Cameron Bruggeman, Jordan Keller, Joe Kramer-Miller, Paul Lewis, Feiqi Jiang, Sebastien Picard, and Mike Wong.

I would like to thank my friends Yaim Cooper and Ruozhou Jia, the MIT Mystery Hunt, and New York's art museums for giving me occasional breaks from my studies.

The University of Chicago Young Scholars Program and Canada/USA Mathcamp provided me with early opportunities to think deeply about mathematics. Mathcamp also played a large part in my decision to switch from physics to number theory. As a physics grad student, I had struggled to find enthusiasm for the sorts of questions that other string theorists were studying. Visiting Mathcamp in 2010 and remembering the many hours I had spent pondering topics like ring theory and topology convinced me that mathematics was where I really belonged.

I would like to thank my physics PhD advisor, Christopher Herzog, for his understanding and support when I decided that I wanted to switch fields. 
I would like to thank Kiran Kedlaya and Shou-Wu Zhang for their guidance when I was an undergraduate and physics $\mathrm{PhD}$ student, respectively.

I enjoyed my time at the Arizona Winter School, and in particular I would like to thank Ana Caraiani and Christian Johansson for organizing a project on infinite level Shimura varieties.

Last, but certainly not least, I would like to thank my parents. They have always been extremely supportive of me; it is hard to imagine where I would be without their support and guidance. 


\section{INTRODUCTION}

The study of $p$-adic families of automorphic forms began with the work of Hida [Hid86, Hid88, Hid94]. Coleman and Mazur [CM98, Col96, Col97] introduced the eigencurve, which parameterizes overconvergent $p$-adic modular forms of finite slope. Coleman and Mazur used a geometric definition of $p$-adic modular forms, based on the original definition of Katz [Kat73]. It is also possible to define $p$-adic automorphic forms using a cohomological approach. Several constructions of eigenvarieties are based on overconvergent cohomology, introduced by Stevens [Ste94] and later generalized by Ash-Stevens AS08]. These include the constructions of Urban [Urb11] and Hansen [Han15]. Emerton Eme06b] has also constructed eigenvarieties using a somewhat different cohomological approach.

The eigenvarieties mentioned above are all rigid analytic spaces, so they parameterize forms that have coefficients in $\mathbb{Q}_{p}$-algebras. Recently, there has been interest in studying forms with coefficients in characteristic $p$. Liu, Wan, and Xiao [LWX17] constructed $\mathbb{Z}_{p} \llbracket \mathbb{Z}_{p}^{\times} \rrbracket$-modules of automorphic forms for definite quaternion algebras. By taking quotients of this module, one can obtain both traditional $p$-adic automorphic forms and forms with coefficients in $\mathbb{F}_{p} \llbracket \mathbb{Z}_{p}^{\times} \rrbracket$ whose existence had been conjectured by Coleman. Using these modules, Liu, Wan, and Xiao proved certain cases of a conjecture of Coleman-Mazur and Buzzard-Kilford BK05 concerning the eigenvalues of the $U_{p}$ operator near the boundary of the weight space. Andreatta, Iovita, and Pilloni AIP constructed an eigencurve that included characteristic $p$ points by extending Katz's definition of $p$-adic modular forms.

In this paper, we will show how Urban's eigenvarieties can be extended to include the characteristic $p$ points at the boundary of weight space. 
In order to explain our results in more detail, we will first describe the basic idea of overconvergent cohomology. Let $G$ be a reductive algebraic group over $\mathbb{Q}$ such that $G\left(\mathbb{Q}_{p}\right)$ is quasisplit. Let $\mathbb{A}$ be the adeles over $\mathbb{Q}$, let $\mathbb{A}_{f}^{p}$ be the finite adeles away from $p$, let $G_{\infty}^{+}$be the identity component of $G(\mathbb{R})$, and let $Z_{G}$ be the center of $G$. Let $T_{0}$ be a maximal compact torus of $G\left(\mathbb{Q}_{p}\right)$, and let $N_{0}^{-}$be an open compact subgroup of a maximal unipotent subgroup of $G\left(\mathbb{Q}_{p}\right)$. We may consider the space

$$
\mathcal{X}:=G(\mathbb{A}) / K^{p} G_{\infty}^{+}
$$

as a locally $\mathbb{Q}_{p}$-analytic manifold. Let $F$ be a finite extension of $\mathbb{Q}_{p}$, and let $\lambda$ : $T_{0} \rightarrow F^{\times}$be a continuous homomorphism. Let $\mathcal{D}_{c, \lambda}$ be the space of compactly supported $F$-valued locally analytic distributions on $\mathcal{X}$, modulo the relations that right translation by $N_{0}^{-}$acts as the identity, right translation by $T_{0}$ acts by $\lambda$, and translation by $Z_{G}(\mathbb{Q})$ acts by the identity. One may think of the cohomology groups $H^{i}\left(G^{\text {ad }}(\mathbb{Q}), \mathcal{D}_{c, \lambda}\right)$ as spaces of $p$-adic automorphic forms. One can also study families of $p$-adic automorphic forms by replacing $F$ with an affinoid $\mathbb{Q}_{p}$-algebra $A$.

There is no need to limit ourselves to $\mathbb{Q}_{p}$-algebras, however. The only real constraint on $A$ is that we must be able to define reasonable notions of locally analytic $A$-valued functions and distributions on $\mathcal{X}$. We will give such a definition when $A$ is a complete Tate $\mathbb{Z}_{p}$-algebra.

To see what the definition should be, we recall a fact from $p$-adic functional analysis: a function $f: \mathbb{Z}_{p} \rightarrow \mathbb{Q}_{p}$ is locally analytic if and only if it is of the form $f(z)=$ $\sum_{n=0}^{\infty} a_{n}\left(\begin{array}{l}z \\ n\end{array}\right)$, where $a_{n} \in A$ and $\left|a_{n}\right|_{p}$ go to zero exponentially as $n \rightarrow \infty$. We will therefore define the space $\mathcal{A}\left(\mathbb{Z}_{p}, A\right)$ of "locally analytic" functions $\mathbb{Z}_{p} \rightarrow A$ to be the set of functions of the form $\sum_{n=0}^{\infty} a_{n}\left(\begin{array}{l}z \\ n\end{array}\right)$, where $a_{n} \in A$ and $a_{n}$ to to zero exponentially (i. e. $\alpha^{-n} a_{n}$ goes to zero for some topologically nilpotent unit $\alpha$ ) as $n \rightarrow \infty$. If $p$ is 
invertible in $A$, then this definition is known to coincide with the usual one. We will make a similar definition for locally analytic functions $\mathbb{Z}_{p}^{k} \rightarrow A$, and then extend the definition to locally $\mathbb{Q}_{p}$-analytic manifolds by gluing.

If $X$ is a locally $\mathbb{Q}_{p}$-analytic manifold, then we will define modules $\mathcal{A}(X, A)$, $\mathcal{D}(X, A), \mathcal{A}_{c}(X, A), \mathcal{D}_{c}(X, A)$ of locally analytic functions, distributions, compactly supported functions, and compactly supported distributions, respectively.

Theorem 1.1. The modules $\mathcal{A}(X, A), \mathcal{D}(X, A), \mathcal{A}_{c}(X, A)$, and $\mathcal{D}_{c}(X, A)$ satisfy the following properties:

(1) $\mathcal{A}(X, A)$ is ring.

(2) If $g: X \rightarrow Y$ is a locally analytic map, then composition with $g$ induces homomorphisms $\mathcal{A}(Y, A) \rightarrow \mathcal{A}(X, A)$ and $\mathcal{D}(X, A) \rightarrow \mathcal{D}(Y, A)$.

(3) The functors $U \mapsto \mathcal{A}(U, A)$ and $U \mapsto \mathcal{D}_{c}(U, A)$ are sheaves on $X$.

(4) If $X$ has the structure of a finitely generated $\mathbb{Z}_{p}$-module, then any continuous group homomorphism $X \rightarrow A^{\times}$is in $\mathcal{A}(X, A)$.

In $\mid$ Urb11], Urban constructed eigenvarieties for reductive groups $G$ such that $G(\mathbb{R})$ has discrete series. We will show how to use the locally analytic distribution modules mentioned above to extend Urban's construction to include characteristic $p$ points.

Theorem 1.2. The reduced eigenvariety constructed in [Urb11] extends to an adic space $\mathcal{E}$ over the weight space $\mathcal{W}=\mathrm{Spa}\left(\mathbb{Z}_{p} \llbracket T^{\prime} \rrbracket, \mathbb{Z}_{p} \llbracket T^{\prime} \rrbracket\right)^{\text {an }}$, where $T^{\prime}$ is a quotient of a compact subgroup of a maximal torus in $G\left(\mathbb{Q}_{p}\right)$. Furthermore, $\mathcal{E}$ is equidimensional and is finite over the spectral variety $\mathcal{Z}$.

We will also correct an error in Urb11]. In order to argue that certain character distributions are uniquely defined, Urban assumed that the region of convergence of 
an Eisenstein series is (up to translation) a union of Weyl chambers. However, this assumption is not true. We will give a new argument for uniqueness.

As this work was being prepared, I became aware that Christian Johansson and James Newton were independently pursuing similar work. In [JN], they adapt Hansen's construction of eigenvarieties to include the boundary of weight space. Their definition of locally analytic distributions on $\mathbb{Z}_{p}^{k}$ is essentially the same as ours. To construct distributions on $p$-adic Lie groups, they use a particular choice of coordinate charts previously studied by Schneider and Teitelbaum. Our definition of locally analytic distributions therefore generalizes theirs.

\section{Modules over Complete Tate Rings}

We will repeat the basic setup of [Buz07, AIP]. Throughout this section, $A$ will denote a complete Tate ring.

\subsection{Definitions.}

Definition 2.1.1. Let $X$ be a quasi-compact topological space, and let $M$ be a topological abelian group. We define $\mathcal{C}(X, M)$ to be the space of continuous functions $X \rightarrow M$, with the topology of uniform convergence.

Definition 2.1.2. Let $S$ be a set, and let $M$ be a topological abelian group. We define $c(S, M)$ to be the space of functions $f: S \rightarrow M$ such that for any open neighborhood $U$ of the identity in $M$, the complement of $f^{-1}(U)$ is finite. We give $c(S, M)$ the topology of uniform convergence.

Definition 2.1.3. Let $M$ be a topological $A$-module. We say that $M$ is orthonormalizable if it is isomorphic to $c(S, A)$ for some set $S$. We say that $M$ is projective if it is a direct summand of an orthonormalizable $A$-module. 
Definition 2.1.4. Let $M$ be a topological $A$-module. We say that a set $B \subset M$ is bounded if for all open neighborhoods $U$ of the identity in $M$, there exists $\alpha \in A^{\times}$so that $\alpha B \subseteq U$.

Definition 2.1.5. Let $M$ and $N$ be topological $A$-modules. We define $\mathcal{L}_{b}(M, N)$ to be the set of continuous $A$-module homomorphisms $M \rightarrow N$, with the topology of convergence on bounded subsets.

Definition 2.1.6. Let $M$ and $N$ be topological $A$-modules. We say that an $A$ module homomorphism $M \rightarrow N$ has has finite rank if its image is a finitely generated $A$-module. We say that an element of $\mathcal{L}_{b}(M, N)$ is completely continuous if it is in the closure of the subspace of finite rank elements.

\subsection{Spectral theory.}

Definition 2.2.1. We define $A\{\{X\}\}$ to be the set of power series $P(X)=\sum_{n=0}^{\infty} a_{n} X^{n}$, $a_{n} \in A$, such that for any $\alpha \in A^{\times}, \alpha^{-n} a_{n} \rightarrow 0$ as $n \rightarrow \infty$.

We say that $P(X) \in A\{\{X\}\}$ is a Fredholm series if it has leading coefficient 1 .

If the adic space $\operatorname{Spa}\left(A, A^{+}\right) \times \mathbb{A}^{1}$ exists, then $A\{\{X\}\}$ is its ring of global sections.

Now assume $A$ is Noetherian. If $M$ is a projective $A$-module and $u: M \rightarrow M$ is completely continuous, then we define the Fredholm series $P(X)=\operatorname{det}(1-X u) \in$ $A\{\{X\}\}$ as in Buz07, AIP.

As in [Urb11], we will need to work with complexes. Let $M^{\bullet}$ be a bounded complex of projective $A$-modules. We will say that $u^{\bullet}: M^{\bullet} \rightarrow M^{\bullet}$ is completely continuous if each $u^{i}$ is completely continuous. If $u^{\bullet}$ is completely continuous, then we define

$$
\operatorname{det}\left(1-X u^{\bullet}\right):=\prod_{\substack{i \\ 5}} \operatorname{det}\left(1-X u^{i}\right)^{(-1)^{i}} .
$$


Lemma 2.2.2. Let $M^{\bullet}$ be a bounded complex of projective A-modules, and let $u^{\bullet}, v^{\bullet}$ : $M^{\bullet} \rightarrow M^{\bullet}$ be completely continuous maps that are homotopy equivalent. Then $\operatorname{det}(1-$ $\left.X u^{\bullet}\right)=\operatorname{det}\left(1-X v^{\bullet}\right)$.

Proof. The coefficients of $\operatorname{det}\left(1-X u^{\bullet}\right)^{-1}$ are the traces of $u^{\bullet}$ acting on the "supersymmetric powers" of $M^{\bullet}$. The $k$ th supersymmetric power of $M^{\bullet}$ consists of formal products of $k$ elements of $M^{\bullet}$, subject to the relation that elements of odd degree anticommute with each other and all other pairs of elements commute. So it suffices to show that $u^{\bullet}$ and $v^{\bullet}$ have the same trace. Then we may use the argument of [Urb11, Lemma 2.2.8].

2.3. Norms. It is often convenient to work with norms on $A$ and on $A$-modules.

Definition 2.3.1. Let $\alpha$ be a topologically nilpotent unit of $A$. We define an $\alpha$ Banach norm on $A$ to be a continuous map $|\cdot|: A \rightarrow \mathbb{R}^{\geq 0}$ satisfying the following conditions.

- $|a+b| \leq \max (|a|,|b|) \quad \forall a, b \in A$

- $|a b| \leq|a||b| \quad \forall a, b \in A$

- $|0|=0,|1|=1,|\alpha|\left|\alpha^{-1}\right|=1$

- The norm $|\cdot|$ induces the topology of $A$.

Definition 2.3.2. Let $\alpha$ be a topologically nilpotent unit of $A$, let $|\cdot|$ be an $\alpha$-Banach norm on $A$, and let $M$ be a topological $A$-module. We define a $|\cdot|$-compatible norm on $M$ to be a continuous map $\|\cdot\|: M \rightarrow \mathbb{R}^{\geq 0}$ satisfying the following conditions.

- $\|m+n\| \leq \max (\|m\|,\|n\|) \quad \forall m, n \in M$

- $\|a m\| \leq|a|\|m\| \quad \forall a \in A, m \in M$

- $\|0\|=0$

If, in addition, $\|\cdot\|$ induces the topology of $M$, we say that $\|\cdot\|$ is a Banach norm. 
For any topologically nilpotent unit $\alpha \in A^{\times}$and ring of definition $A_{0}$ of $A$ containing $\alpha$, the function $|\cdot|: A \rightarrow \mathbb{R}^{\geq 0}$ defined by

$$
|a|=\inf _{n \in \mathbb{Z} \mid \alpha^{n} a \in A_{0}} p^{n}
$$

is an $\alpha$-Banach norm.

Furthermore, if $M$ is a topological $A$-module and $M_{0}$ is an open neighborhood of zero in $M$ that is an $A_{0}$-module, then the function $\|\cdot\|: M \rightarrow \mathbb{R}^{\geq 0}$ defined by

$$
\|m\|=\inf _{n \in \mathbb{Z} \mid \alpha^{n} m \in M_{0}} p^{n}
$$

is a norm compatible with $|\cdot|$. If the sets of the form $\alpha^{n} M_{0}$ are a basis of open neighborhoods of zero, then this norm is Banach.

\section{LOCALLY ANALYTIC FUNCTIONS AND DISTRIBUTIONS}

Now let $A$ be a complete Tate $\mathbb{Z}_{p}$-algebra, and let $X$ be a locally $\mathbb{Q}_{p}$-analytic manifold. In this section, we will define modules $\mathcal{A}(X, A)$ and $\mathcal{D}(X, A)$ of "locally analytic" $A$-valued functions and distributions on $X$.

The space $X$ can be covered by coordinate patches isomorphic to $\mathbb{Z}_{p}^{k}$ for some $k$. We will first define locally analytic functions on these patches and then show that the construction can be glued.

3.1. Preliminaries. We will recall some basic facts about $p$-adic functional analysis.

We will make use of the completed group ring $\mathbb{Z}_{p} \llbracket \mathbb{Z}_{p}^{k} \rrbracket=\lim _{n} \mathbb{Z}_{p}\left[\mathbb{Z}_{p}^{k} / p^{n} \mathbb{Z}_{p}^{k}\right]$.

For $z \in \mathbb{Z}_{p}^{k}$, let $[z]$ denote the corresponding element of $\mathbb{Z}_{p} \llbracket \mathbb{Z}_{p}^{k} \rrbracket$, and let $\Delta_{z}=[z]-[0]$. Let $I_{\Delta}$ denote the augmentation ideal of $\mathbb{Z}_{p} \llbracket \mathbb{Z}_{p}^{k} \rrbracket$; this is the ideal generated by the $\Delta_{v}$. The maximal ideal of $\mathbb{Z}_{p} \llbracket \mathbb{Z}_{p}^{k} \rrbracket$ is $(p)+I_{\Delta}$. 
We let $\mathbb{Z}_{p}^{k}$ act on $\mathcal{C}\left(\mathbb{Z}_{p}^{k}, M\right)$ by translation: for $g \in \mathcal{C}\left(\mathbb{Z}_{p}^{k}, M\right),(z g)(y)=g(y+z)$. This action extends to an action of $\mathbb{Z}_{p} \llbracket \mathbb{Z}_{p}^{k} \rrbracket$.

To simplify notation, if $z=\left(z_{1}, \ldots, z_{k}\right) \in \mathbb{Z}_{p}^{k}$, and $n=\left(n_{1}, \ldots, n_{k}\right) \in \mathbb{N}^{k}$, we will write $\left(\begin{array}{c}z \\ n\end{array}\right)$ for $\prod_{i=1}^{k}\left(\begin{array}{c}z_{i} \\ n_{i}\end{array}\right)$, and we will write $\sum n$ for $\sum_{i=1}^{k} n_{i}$.

Lemma 3.1.1 (Mahler's theorem, Laz65, Théorème II.1.2.4]). Let $M$ be a complete topological $\mathbb{Z}_{p}$-module. Suppose that $M$ has a basis of open neighborhoods of zero that are subgroups of $M$. There is an isomorphism $c\left(\mathbb{N}^{k}, M\right) \stackrel{\sim}{\rightarrow} \mathcal{C}\left(\mathbb{Z}_{p}^{k}, M\right)$ that sends $f \in c\left(\mathbb{N}^{k}, M\right)$ to a function $g \in \mathcal{C}\left(\mathbb{Z}_{p}^{k}, M\right)$ defined by

$$
g(z)=\sum_{n \in \mathbb{N}^{k}} f(n)\left(\begin{array}{l}
z \\
n
\end{array}\right) .
$$

We say that the right-hand side of the above equation is the Mahler expansion of $g$.

Lemma 3.1.2 (Amice's theorem). Let $F$ be a closed subfield of $\mathbb{C}_{p}$, and let $L A_{h}\left(\mathbb{Z}_{p}^{k}, F\right)$ be the space of functions $\mathbb{Z}_{p}^{k} \rightarrow F$ that extend to an analytic function $\mathbb{Z}_{p}^{k}+p^{h} \mathcal{O}_{\mathbb{C}_{p}}^{k} \rightarrow \mathbb{C}_{p}$. For $f \in L A_{h}\left(\mathbb{Z}_{p}^{k}, F\right)$, define

$$
|f|:=\sup _{z \in \mathbb{Z}_{p}^{k}+p^{h} \mathcal{O}_{\mathbb{C}_{p}}^{k}}|f(z)|_{p}
$$

Then the functions $\left\lfloor\frac{n_{1}}{p^{h}}\right\rfloor ! \ldots\left\lfloor\frac{n_{k}}{p^{h}}\right\rfloor !\left(\begin{array}{c}z \\ n\end{array}\right)$ form an orthonormal basis for the Banach space $L A_{h}\left(\mathbb{Z}_{p}^{k}, F\right)$. In other words, every $f \in L A_{h}\left(\mathbb{Z}_{p}^{k}, F\right)$ can be expressed uniquely in the form

$$
f(z)=\sum_{n \in \mathbb{N}^{k}} a_{n}\left\lfloor\frac{n_{1}}{p^{h}}\right\rfloor ! \ldots\left\lfloor\frac{n_{k}}{p^{h}}\right\rfloor !\left(\begin{array}{c}
z \\
n
\end{array}\right),
$$

and $|f|=\sup _{n \in \mathbb{N}^{k}}\left|a_{n}\right|_{p}$.

Proof. This follows from Ami64, Chapitre 3] (see also [Col10, Théorème I.4.7]). 
The following formulas concerning the $p$-adic valuations of $n$ !, where $n$ is a nonnegative integer, are well-known.

$$
\begin{gathered}
v_{p}(n !)=\sum_{k=1}^{\infty}\left\lfloor\frac{n}{p^{k}}\right\rfloor \\
\frac{n}{p-1}-\log _{p}(n+1) \leq v_{p}(n !) \leq \frac{n}{p-1}
\end{gathered}
$$

Consequently, if $F$ is a closed subfield of $\mathbb{C}_{p}$, and $f: \mathbb{Z}_{p}^{k} \rightarrow F$ is a continuous function with the Mahler expansion $f(z)=\sum_{n \in \mathbb{N}^{k}} a_{n}\left(\begin{array}{l}z \\ n\end{array}\right)$, then $f$ is locally analytic if and only if $\left|a_{n}\right|_{p}$ go to zero exponentially in $\sum n$.

3.2. Definitions. The above facts suggest that we should define a function $\mathbb{Z}_{p}^{k} \rightarrow A$ to be "locally analytic" if the coefficients of its Mahler expansion decrease to zero exponentially.

We choose a topologically nilpotent $\alpha \in A^{\times}$.

Definition 3.2.1. Let $r \in \mathbb{R}^{+}$. We define $\mathcal{A}^{(\alpha, r)}\left(\mathbb{Z}_{p}^{k}, A\right)$ to be the space of functions $f \in \mathcal{C}\left(\mathbb{Z}_{p}^{k}, A\right)$ such that for any open neighborhood $U$ of zero in $A$, there exists $N \in \mathbb{N}$ so that for all integers $n>N$ and all $\delta \in I_{\Delta}^{n}, \alpha^{\lfloor-r n\rfloor} \delta f \in \mathcal{C}\left(\mathbb{Z}_{p}^{k}, U\right)$.

For any open neighborhood $U$ of zero in $A$, we define $U_{r} \subset \mathcal{A}^{(\alpha, r)}\left(\mathbb{Z}_{p}^{k}, A\right)$ to be the set of all $f \in \mathcal{A}^{(\alpha, r)}\left(\mathbb{Z}_{p}^{k}, A\right)$ such that $\alpha^{\lfloor-r n\rfloor} \delta f \in \mathcal{C}\left(\mathbb{Z}_{p}^{k}, U\right)$ for all $n \in \mathbb{N}$ and all $\delta \in I_{\Delta}^{n}$. We define a topology on $\mathcal{A}^{(\alpha, r)}(U, A)$ by making sets of the form $U_{r}$ a basis of open neighborhoods of zero.

We define $\mathcal{A}\left(\mathbb{Z}_{p}^{k}, A\right)=\lim _{\longrightarrow} \mathcal{A}^{(\alpha, r)}\left(\mathbb{Z}_{p}^{k}, A\right)$.

The connection between this definition and Mahler expansions will be explained by Lemma 3.2.3.

The definition of $\mathcal{A}^{(\alpha, r)}\left(\mathbb{Z}_{p}^{k}, A\right)$ is invariant under affine changes of coordinates. 
For any topologically nilpotent unit $\alpha^{\prime} \in A^{\times}$and sufficiently small $r^{\prime} \in \mathbb{R}^{+}$, $\mathcal{A}^{\left(\alpha^{\prime}, r^{\prime}\right)}\left(\mathbb{Z}_{p}^{k}, A\right)$ injects into $\mathcal{A}^{(\alpha, r)}\left(\mathbb{Z}_{p}^{k}, A\right)$. So the directed systems $\left(\mathcal{A}^{(\alpha, r)}\left(\mathbb{Z}_{p}^{k}, A\right)\right)_{r \in \mathbb{R}^{+}}$ and $\left(\mathcal{A}^{\left(\alpha^{\prime}, r\right)}\left(\mathbb{Z}_{p}^{k}, A\right)\right)_{r \in \mathbb{R}^{+}}$are cofinal, and $\mathcal{A}\left(\mathbb{Z}_{p}^{k}, A\right)$ does not depend on the choice of $\alpha$. If $F$ is a closed subfield of $\mathbb{C}_{p}$, then there are continuous injections with dense image

$$
\mathcal{A}^{\left(p, 1 /(p-1) p^{h}\right)}\left(\mathbb{Z}_{p}^{k}, F\right) \hookrightarrow L A_{h}\left(\mathbb{Z}_{p}^{k}, F\right) \hookrightarrow \mathcal{A}^{(p, r)}\left(\mathbb{Z}_{p}^{k}, F\right)
$$

for any $r<\frac{1}{(p-1) p^{h}}$, so the directed systems $\left(\mathcal{A}^{(p, r)}\left(\mathbb{Z}_{p}^{k}, F\right)\right)_{r \in \mathbb{R}^{+}}$and $\left(L A_{h}\left(\mathbb{Z}_{p}^{k}, F\right)\right)_{h \in \mathbb{N}}$ are also cofinal.

The module $\mathcal{A}^{(\alpha, r)}\left(\mathbb{Z}_{p}^{k}, A\right)$ can also be defined (albeit less symmetrically) using $\alpha$ Banach norms. Choose a ring of definition $A_{0}$ of $A$, and define an $\alpha$-Banach norm $|\cdot|: A \rightarrow \mathbb{R}^{\geq 0}$ as in section 2.3 . Define $\|\cdot\|_{0}: \mathcal{C}\left(\mathbb{Z}_{p}^{k}, A\right) \rightarrow \mathbb{R}^{\geq 0}$ by

$$
\|f\|_{0}=\sup _{z \in \mathbb{Z}_{p}^{k}}|f(z)|
$$

Then

$$
\mathcal{A}^{(\alpha, r)}\left(\mathbb{Z}_{p}^{k}, A\right)=\left\{\left.f \in \mathcal{C}\left(\mathbb{Z}_{p}^{k}, A\right)\left|\limsup _{n \rightarrow \infty} \sup _{\delta \in I_{\Delta}^{n}}\right| \alpha^{\lfloor-r n\rfloor} \delta f\right|_{0}=0\right\},
$$

and we define $\|\cdot\|_{r}: \mathcal{A}^{(\alpha, r)}\left(\mathbb{Z}_{p}^{k}, A\right) \rightarrow \mathbb{R}^{\geq 0}$ by

$$
\|f\|_{r}=\sup _{n \in \mathbb{N}} \sup _{\delta \in I_{\Delta}^{n}}\left\|\alpha^{\lfloor-r n\rfloor} \delta f\right\|_{0} .
$$

The functions $\|\cdot\|_{0}$ and $\|\cdot\|_{r}$ are Banach norms compatible with $|\cdot|$.

Presumably, it would be reasonable to define $\mathcal{A}^{(\alpha, r)}\left(\mathbb{Z}_{p}^{k}, M\right)$ and $\mathcal{A}\left(\mathbb{Z}_{p}^{k}, M\right)$ for any topological $A$-module $M$ that is locally convex in the sense that for some (equivalently, any) ring of definition $A_{0}$ of $A, M$ has a basis of open neighborhoods of the identity that are $A_{0}$-modules. (We would just replace $A$ with $M$ in the above definition.) However, we will not need this additional generality. 
Definition 3.2.2. Let $r \in \mathbb{R}^{+}$. We define $\mathcal{D}^{(\alpha, r)}\left(\mathbb{Z}_{p}^{k}, A\right)$ to be the closure of the image of $\mathcal{L}_{b}\left(\mathcal{C}\left(\mathbb{Z}_{p}^{k}, A\right), A\right)$ in $\mathcal{L}_{b}\left(\mathcal{A}^{(\alpha, r)}\left(\mathbb{Z}_{p}^{k}, A\right), A\right)$.

We define $\mathcal{D}(X, A)=\lim _{\leftarrow} \mathcal{D}^{(\alpha, r)}(X, A)$.

The definition of $\mathcal{D}(X, A)$ does not depend on the choice of $\alpha$.

We chose the definitions of $\mathcal{A}^{(\alpha, r)}(X, A)$ and $\mathcal{D}^{(\alpha, r)}(X, A)$ so that these modules would be orthonormalizable, as we will now show.

Lemma 3.2.3. There is an isomorphism Ser : $c\left(\mathbb{N}^{k}, A\right) \stackrel{\sim}{\rightarrow} \mathcal{A}^{(\alpha, r)}\left(\mathbb{Z}_{p}^{k}, A\right)$ that sends $f \in c\left(\mathbb{N}^{k}, A\right)$ to a function $g \in \mathcal{A}^{(\alpha, r)}\left(\mathbb{Z}_{p}^{k}, A\right)$ defined by

$$
g(z)=\sum_{n \in \mathbb{N}^{k}} \alpha^{\left\lceil r \sum n\right\rceil} f(n)\left(\begin{array}{l}
z \\
n
\end{array}\right) .
$$

There is an isomorphism $\mathrm{Ev}: \mathcal{D}^{(\alpha, r)}\left(\mathbb{Z}_{p}^{k}, A\right) \stackrel{\sim}{\rightarrow} c\left(\mathbb{N}^{k}, A\right)$ that sends $\phi \in \mathcal{D}^{(\alpha, r)}\left(\mathbb{Z}_{p}^{k}, A\right)$ to a function $f \in c\left(\mathbb{N}^{k}, A\right)$ defined by

$$
f(n)=\alpha^{\left\lceil r \sum n\right\rceil} \phi\left(\left(\begin{array}{l}
z \\
n
\end{array}\right)\right) .
$$

Hence $\mathcal{A}^{(\alpha, r)}(X, A)$ and $\mathcal{D}^{(\alpha, r)}(X, A)$ are orthonormalizable.

Proof. Let $f \in c\left(\mathbb{N}^{k}, A\right)$, and let $g$ be defined by 3.2.4. By Mahler's theorem, $g \in \mathcal{C}(X, A)$. We observe that for any $h \in \mathcal{C}(X, A)$ and $\delta \in \mathbb{Z}_{p} \llbracket \mathbb{Z}_{p}^{k} \rrbracket,\|\delta h\|_{0} \leq\|h\|_{0}$. Furthermore, if $\delta \in I_{\Delta}^{m}$, then $\delta\left(\begin{array}{l}z \\ n\end{array}\right)=0$ whenever $\sum n<m$. So

$$
\begin{aligned}
\left\|\alpha^{\lfloor-r m\rfloor} \delta g\right\|_{0} & \leq \sup _{\sum n \geq m}\left|\alpha^{\lfloor-r m\rfloor+\left\lceil r \sum n\right\rceil} f(n)\right| \\
& \leq \sup _{\sum n \geq m}|f(n)| .
\end{aligned}
$$

It follows that $g \in \mathcal{A}^{(\alpha, r)}\left(\mathbb{Z}_{p}^{k}, A\right)$, and Ser is continuous. 
We can recover $f$ from $g$ using the relation

$$
f(n)=\alpha^{\left\lfloor-r \sum n\right\rfloor}\left(\Delta_{e_{1}}^{n_{1}} \ldots \Delta_{e_{k}}^{n_{k}} g\right)(0)
$$

where $e_{1}, \ldots, e_{k}$ are the standard basis for $\mathbb{Z}_{p}^{k}$. Since $|f(n)| \leq \sup _{\delta \in\left(I_{\Delta}\right) \sum n}\|\delta g\|_{0}$, the above relation induces a continuous map Coeff $: \mathcal{A}^{(\alpha, r)}\left(\mathbb{Z}_{p}^{k}, A\right) \rightarrow c\left(\mathbb{Z}_{p}^{k}, A\right)$ that is a left inverse of Ser. To see that Coeff is also a right inverse of Ser, observe that $($ Ser $\circ$ Coeff $)(g)$ and $g$ agree on $\mathbb{N}^{k}$, which is dense in $\mathbb{Z}_{p}^{k}$.

The map Ser induces an isomorphism $\operatorname{Ser}^{*}: \mathcal{L}_{b}\left(\mathcal{A}^{(\alpha, r)}\left(\mathbb{Z}_{p}^{k}, A\right), A\right) \stackrel{\sim}{\rightarrow} \mathcal{L}_{b}\left(c\left(\mathbb{N}^{k}, A\right), A\right)$. The pairing $c\left(\mathbb{N}^{k}, A\right) \times c\left(\mathbb{N}^{k}, A\right) \rightarrow A$ defined by $(f, g) \mapsto \sum_{n \in \mathbb{N}^{k}} f(n) g(n)$ identifies $c\left(\mathbb{N}^{k}, A\right)$ with a submodule of $\mathcal{L}_{b}\left(c\left(\mathbb{N}^{k}, A\right), A\right)$. For any $\phi \in \mathcal{L}_{b}\left(\mathcal{C}\left(\mathbb{Z}_{p}^{k}, A\right), A\right)$, the function $n \mapsto \phi\left(\left(\begin{array}{l}z \\ n\end{array}\right)\right)$ is bounded, so in particular $\alpha^{\left\lceil r \sum n\right\rceil} \phi\left(\left(\begin{array}{l}z \\ n\end{array}\right)\right) \rightarrow 0$ as $\sum n \rightarrow \infty$. Hence the image of Ser* is contained in $c\left(\mathbb{N}^{k}, A\right)$. Furthermore, the image contains all elements of $c\left(\mathbb{N}^{k}, A\right)$ that are supported on a finite subset of $\mathbb{N}^{k}$, and these elements are dense in $c\left(\mathbb{N}^{k}, A\right)$.

Lemma 3.2.3 makes it clear that for $r^{\prime}<r$, there are natural injections

$$
\begin{aligned}
& \mathcal{D}^{\left(\alpha, r^{\prime}\right)}\left(\mathbb{Z}_{p}^{k}, A\right) \hookrightarrow \mathcal{L}_{b}\left(\mathcal{A}^{\left(\alpha, r^{\prime}\right)}\left(\mathbb{Z}_{p}^{k}, A\right), A\right) \hookrightarrow \mathcal{D}^{(\alpha, r)}\left(\mathbb{Z}_{p}^{k}, A\right) \\
& \mathcal{A}^{(\alpha, r)}\left(\mathbb{Z}_{p}^{k}, A\right) \hookrightarrow \mathcal{L}_{b}\left(\mathcal{D}^{(\alpha, r)}\left(\mathbb{Z}_{p}^{k}, A\right), A\right) \hookrightarrow \mathcal{A}^{\left(\alpha, r^{\prime}\right)}\left(\mathbb{Z}_{p}^{k}, A\right) .
\end{aligned}
$$

3.3. Properties of locally analytic functions and distributions. In this section, we check that $\mathcal{A}^{(\alpha, r)}\left(\mathbb{Z}_{p}^{k}, A\right)$ has some properties that one would expect of locally analytic functions.

Lemma 3.3.1. Multiplication induces a continuous map $\mathcal{A}^{(\alpha, r)}\left(\mathbb{Z}_{p}^{k}, A\right) \times \mathcal{A}^{(\alpha, r)}\left(\mathbb{Z}_{p}^{k}, A\right) \rightarrow$ $\mathcal{A}^{(\alpha, r)}\left(\mathbb{Z}_{p}^{k}, A\right)$. 
Proof. This follows Lemma 3.2.3 and the fact that for $m, n \in \mathbb{N},\left(\begin{array}{c}z \\ n\end{array}\right)\left(\begin{array}{c}z \\ m\end{array}\right)$ is of the form $\sum_{i=0}^{m+n} a_{i}\left(\begin{array}{c}z \\ i\end{array}\right)$ with $a_{i} \in \mathbb{Z}$

Lemma 3.3.2. Let

$$
f: \mathcal{C}\left(\mathbb{Z}_{p}^{k}, \mathbb{Z}_{p}\right) \rightarrow \mathcal{C}\left(\mathbb{Z}_{p}^{j}, \mathbb{Z}_{p}\right)
$$

be a homomorphism of $\mathbb{Z}_{p}$-modules. For any complete Tate ring $A$ and any $r, s \in \mathbb{R}^{+}$, there is at most one continuous A-linear homomorphism $\tilde{f}$ making the diagram

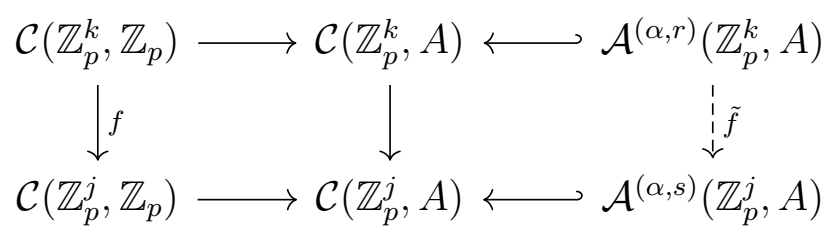

commute, and there is at most one continuous A-linear homomorphism $\tilde{f}^{*}$ making the diagram

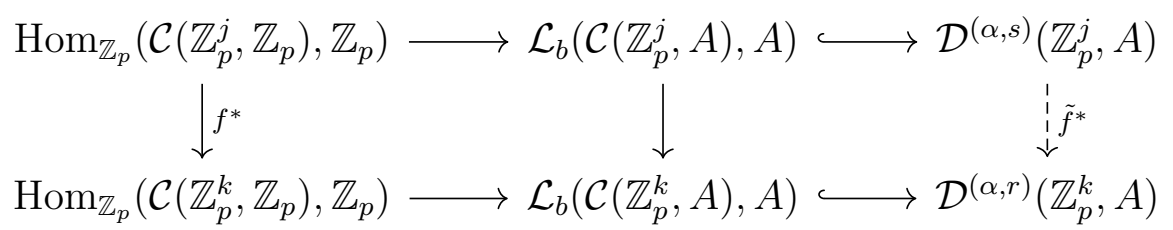

commute. If either homomorphism exists, we say that it is induced by $f$.

Let $A$ be a complete Tate ring, and let $\alpha \in A^{\times}$be a topologically nilpotent unit. There exists $t_{0} \in \mathbb{R}^{+}$so that for any $r, s \in \mathbb{R}^{+}$and any $\mathbb{Z}_{p}$-module homomorphism $f: \mathcal{C}\left(\mathbb{Z}_{p}^{k}, \mathbb{Z}_{p}\right) \rightarrow \mathcal{C}\left(\mathbb{Z}_{p}^{k}, \mathbb{Z}_{p}\right)$ that induces a continuous homomorphism

$$
\mathcal{A}^{(p, r)}\left(\mathbb{Z}_{p}^{k}, \mathbb{Q}_{p}\right) \rightarrow \mathcal{A}^{(p, s)}\left(\mathbb{Z}_{p}^{j}, \mathbb{Q}_{p}\right)
$$

$f$ also induces continuous homomorphisms

$$
\begin{gathered}
\mathcal{A}^{(\alpha, r t)}\left(\mathbb{Z}_{p}^{k}, A\right) \rightarrow \mathcal{A}^{(\alpha, s t)}\left(\mathbb{Z}_{p}^{j}, A\right) \\
\mathcal{D}^{(\alpha, s t)}\left(\mathbb{Z}_{p}^{j}, A\right) \rightarrow \mathcal{D}^{(\alpha, r t)}\left(\mathbb{Z}_{p}^{k}, A\right)
\end{gathered}
$$

for all $t \in\left(0, t_{0}\right)$. 
Proof. We look at the matrix coefficients of $f$ in the basis of Lemma 3.2.3. Write

$$
f\left(\left(\begin{array}{l}
z \\
n
\end{array}\right)\right)=\sum_{m \in \mathbb{Z}_{p}^{j}} a_{n m}\left(\begin{array}{c}
z \\
m
\end{array}\right)
$$

with $a_{n m} \in \mathbb{Z}_{p}$. If the restriction $\mathcal{A}^{(\alpha, r t)}\left(\mathbb{Z}_{p}^{k}, A\right) \rightarrow \mathcal{A}^{(\alpha, s t)}\left(\mathbb{Z}_{p}^{j}, A\right)$ exists, its matrix must have entries $\alpha^{\left\lfloor\left(r \sum n-s \sum m\right) t\right\rfloor} a_{n m}$. Such a matrix defines a continuous map if and only if the following two conditions are satisfied.

(1) $\alpha^{\left\lfloor\left(r \sum n-s \sum m\right) t\right\rfloor} a_{n m}$ are bounded.

(2) For any fixed $n, \alpha^{\left\lfloor\left(r \sum n-s \sum m\right) t\right\rfloor} a_{n m} \rightarrow 0$ as $\sum m \rightarrow \infty$.

The terms with $r \sum n-s \sum m \geq 0$ are certainly bounded, so we only need to worry about terms with $r \sum n-s \sum m<0$. There exists a positive integer $\ell$ so that $p^{\ell} / \alpha$ is power bounded. If the $p^{\left\lfloor\left(r \sum n-s \sum m\right) t \ell\right\rfloor} a_{n m}$ are bounded (resp. go to zero as $\left.\sum m \rightarrow \infty\right)$, then the same will be true of $\alpha^{\left\lfloor\left(r \sum n-s \sum m\right) t\right\rfloor} a_{n m}$. So we may take $t_{0}=\ell^{-1}$.

The map $\mathcal{D}^{(\alpha, s t)}\left(\mathbb{Z}_{p}^{j}, A\right) \rightarrow \mathcal{D}^{(\alpha, r t)}\left(\mathbb{Z}_{p}^{k}, A\right)$ exists if and only if condition (1) above and the following condition are satisfied.

$\left(2^{\prime}\right)$ For any fixed $m, \alpha^{\left\lfloor\left(r \sum n-s \sum m\right) t\right\rfloor} a_{n m} \rightarrow 0$ as $\sum n \rightarrow \infty$.

Since $a_{n m} \in \mathbb{Z}_{p}$ and $\alpha^{\left\lfloor r t \sum n\right\rfloor} \rightarrow 0$ as $\sum n \rightarrow \infty,\left(2^{\prime}\right)$ will always be satisfied.

Proposition 3.3.3. Let $g: \mathbb{Z}_{p}^{j} \rightarrow \mathbb{Z}_{p}^{k}$ be a (globally) analytic function. For some $r_{0} \in \mathbb{R}^{+}$depending on $\alpha$ but not on $g, j, k$, composition with $g$ induces continuous A-linear homomorphisms

$$
\begin{aligned}
\mathcal{A}^{(\alpha, r)}\left(\mathbb{Z}_{p}^{k}, A\right) & \rightarrow \mathcal{A}^{(\alpha, s)}\left(\mathbb{Z}_{p}^{j}, A\right) \\
\mathcal{D}^{(\alpha, s)}\left(\mathbb{Z}_{p}^{j}, A\right) & \rightarrow \mathcal{D}^{(\alpha, r)}\left(\mathbb{Z}_{p}^{k}, A\right)
\end{aligned}
$$

for all $s<r<r_{0}$. 
Proof. There are continuous maps

$$
\mathcal{A}^{(p, 1 /(p-1))}\left(\mathbb{Z}_{p}^{k}, \mathbb{Q}_{p}\right) \rightarrow L A_{0}\left(\mathbb{Z}_{p}^{k}, \mathbb{Q}_{p}\right) \stackrel{g^{*}}{\rightarrow} L A_{0}\left(\mathbb{Z}_{p}^{j}, \mathbb{Q}_{p}\right) \rightarrow \mathcal{A}^{(p, 1 /(p-1)-\epsilon)}\left(\mathbb{Z}_{p}^{j}, \mathbb{Q}_{p}\right)
$$

for any $\epsilon \in(0,1 /(p-1))$. Applying Lemma 3.3.2 then yields the desired result.

If $j=1$, then the maps exist even if $r=s$. We do not know if the same is true for $j>1$. Essentially, when $j=1$, one can prove existence using norms on $L A_{0}$ along with the fact that $v_{p}(n !)-\sum_{i=1}^{k} v_{p}\left(m_{i} !\right) \geq\left\lfloor\left(n-\sum m\right) / p\right\rfloor$. (The same idea will be used in the proof of Proposition 3.3.4.) However, for $j>1, \sum_{i=1}^{j} v_{p}\left(n_{i} !\right)-\sum_{i=1}^{k} v_{p}\left(m_{i} !\right)$ can be zero for arbitrarily large values of $\sum n-\sum m$.

Proposition 3.3.4. Let $S$ be a set of coset representatives of $\mathbb{Z}_{p}^{k} / p \mathbb{Z}_{p}^{k}$. The homeomorphism $\mathbb{Z}_{p}^{k} \times S \stackrel{\sim}{\rightarrow} \mathbb{Z}_{p}^{k}$ defined by $(z, s) \mapsto p z+s$ determines an isomorphism

$$
\mathcal{C}\left(\mathbb{Z}_{p}^{k}, A\right) \cong \mathcal{C}\left(\mathbb{Z}_{p}^{k}, A\right)^{\oplus p^{k}}
$$

which induces isomorphisms

$$
\begin{aligned}
& \mathcal{A}^{(\alpha, r)}\left(\mathbb{Z}_{p}^{k}, A\right) \cong \mathcal{A}^{(\alpha, p r)}\left(\mathbb{Z}_{p}^{k}, A\right)^{\oplus p^{k}} \\
& \mathcal{D}^{(\alpha, r)}\left(\mathbb{Z}_{p}^{k}, A\right) \cong \mathcal{D}^{(\alpha, p r)}\left(\mathbb{Z}_{p}^{k}, A\right)^{\oplus p^{k}}
\end{aligned}
$$

for all sufficiently small $r \in \mathbb{R}^{+}$.

Proof. We will again apply Lemma 3.3.2. As multiplication by $p$ does not mix coordinates, it is sufficient to check the case $k=1$. It is then enough to check that composition with the function

$$
g(z)=p z
$$


defines a continuous homomorphism

$$
\mathcal{A}^{\left(p, 1 / 2 p^{2}\right)}\left(\mathbb{Z}_{p}, \mathbb{Q}_{p}\right) \rightarrow \mathcal{A}^{(p, 1 / 2 p)}\left(\mathbb{Z}_{p}, \mathbb{Q}_{p}\right)
$$

and that composition with the function

$$
h(z)= \begin{cases}z / p, & z \in p \mathbb{Z}_{p} \\ 0, & z \in \mathbb{Z}_{p}^{\times}\end{cases}
$$

defines a continuous homomorphism

$$
\mathcal{A}^{(p, 1 / 2 p)}\left(\mathbb{Z}_{p}, \mathbb{Q}_{p}\right) \rightarrow \mathcal{A}^{\left(p, 1 / 2 p^{2}\right)}\left(\mathbb{Z}_{p}, \mathbb{Q}_{p}\right)
$$

Define $a_{n m}, b_{n m}$ by

$$
g\left(\left(\begin{array}{l}
z \\
n
\end{array}\right)\right)=\sum_{m=0}^{\infty} a_{n m}\left(\begin{array}{c}
z \\
m
\end{array}\right), \quad h\left(\left(\begin{array}{l}
z \\
n
\end{array}\right)\right)=\sum_{m=0}^{\infty} b_{n m}\left(\begin{array}{c}
z \\
m
\end{array}\right) .
$$

We just need to verify that:

(1) $v_{p}\left(a_{n m}\right)-\frac{m}{2 p}+\frac{n}{2 p^{2}}$ is bounded below for $p m \geq n$.

(2) For any $n, v_{p}\left(a_{n m}\right)-\frac{m}{2 p}+\frac{n}{2 p^{2}} \rightarrow \infty$ as $m \rightarrow \infty$.

(3) $v_{p}\left(b_{n m}\right)-\frac{m}{2 p^{2}}+\frac{n}{2 p}$ is bounded below for $m \geq p n$.

(4) For any $n, v_{p}\left(b_{n m}\right)-\frac{m}{2 p^{2}}+\frac{n}{2 p} \rightarrow \infty$ as $m \rightarrow \infty$.

Applying Lemma 3.1 .2 gives

$$
v_{p}\left(a_{n m}\right)-v_{p}(m !) \geq-v_{p}(\lfloor n / p\rfloor !) .
$$

For $n \geq p m$ this implies

$$
v_{p}\left(a_{n m}\right) \geq \sum_{i=1}^{\infty}\left(\left\lfloor m / p^{i}\right\rfloor-\left\lfloor n / p^{i+1}\right\rfloor\right) \geq\left\lfloor m / p-n / p^{2}\right\rfloor .
$$


Similarly, Lemma 3.1 .2 implies

$$
v_{p}\left(b_{n m}\right) \geq \sum_{i=1}^{\infty}\left(\left\lfloor m / p^{i+1}\right\rfloor-\left\lfloor n / p^{i}\right\rfloor\right) \geq\left\lfloor m / p^{2}-n / p\right\rfloor .
$$

Lemma 3.3.5. Any continuous homomorphism $\lambda: \mathbb{Z}_{p}^{k} \rightarrow A^{\times}$is in $\mathcal{A}\left(\mathbb{Z}_{p}^{k}, A\right)$.

Proof. Lemma 3.3.1 allows us to reduce to the one-dimensional case, and Proposition 3.3.4 allows us to replace $\mathbb{Z}_{p}^{k}$ with an open sub-lattice. So it suffices to consider the case where $k=1$ and $(\lambda(1)-1) / \alpha$ is topologically nilpotent. In that case, since

$$
\lambda(z)=\sum_{n=0}^{\infty}\left(\begin{array}{l}
z \\
n
\end{array}\right)(\lambda(1)-1)^{n}
$$

$\lambda \in \mathcal{A}^{(\alpha, 1)}\left(\mathbb{Z}_{p}, A\right)$.

Lemma 3.3.6. For any $0<s<r$, the inclusions $\mathcal{A}^{(\alpha, r)}\left(\mathbb{Z}_{p}^{k}, A\right) \hookrightarrow \mathcal{A}^{(\alpha, s)}\left(\mathbb{Z}_{p}^{k}, A\right)$ and $\mathcal{D}^{(\alpha, s)} \hookrightarrow \mathcal{D}^{(\alpha, r)}\left(\mathbb{Z}_{p}^{k}, A\right)$ are completely continuous.

Proof. In the orthonormal bases of Lemma 3.2.3, these inclusions are represented by diagonal matrices with diagonal entries of the form $\alpha^{\left\lfloor r \sum n\right\rfloor-\left\lfloor s \sum n\right\rfloor}$. As $\sum n \rightarrow \infty$, the entries go to zero.

3.4. Gluing. Propositions 3.3.3 and 3.3.4 show that it makes sense to define locally analytic functions and distributions on arbitrary locally $\mathbb{Q}_{p}$-analytic manifolds by gluing.

Definition 3.4.1. Let $k$ be a nonnegative integer, and let $X$ be a locally $\mathbb{Q}_{p}$-analytic manifold of dimension $k$. Choose a decomposition $X=\bigsqcup_{i \in I} X_{i}$ for some index set $I$, 
and choose an identification of each $X_{i}$ with $\mathbb{Z}_{p}^{k}$. We define

$$
\begin{aligned}
\mathcal{A}(X, A) & =\prod_{i \in I} \mathcal{A}\left(X_{i}, A\right) \\
\mathcal{A}_{c}(X, A) & =\bigoplus_{i \in I} \mathcal{A}\left(X_{i}, A\right) \\
\mathcal{D}(X, A) & =\bigoplus_{i \in I} \mathcal{D}\left(X_{i}, A\right) \\
\mathcal{D}_{c}(X, A) & =\prod_{i \in I} \mathcal{D}\left(X_{i}, A\right) .
\end{aligned}
$$

By Propositions 3.3.3 and 3.3.4, the above definitions do not depend on the choice of decomposition, and the functors $U \mapsto \mathcal{A}(U, A)$ and $U \mapsto \mathcal{D}_{c}(U, A)$ are sheaves on $X$.

3.5. Geometric interpretation of distributions. Suppose that the Tate algebra $A\left\langle T_{1}, \ldots, T_{n}\right\rangle$ is sheafy for each nonnegative integer $n$. The modules of locally analytic distributions have an alternative interpretation as rings of sections of adic spaces. This interpretation will not be used elsewhere in the paper, but it gives further evidence that our definition of distributions is reasonable.

Let $D=\operatorname{Spa}\left(\mathbb{Z}_{p} \llbracket \mathbb{Z}_{p}^{k} \rrbracket, \mathbb{Z}_{p} \llbracket \mathbb{Z}_{p}^{k} \rrbracket\right)$. Let $A^{+}$be an open and integrally closed subring of $A$. Let $Y=D \times_{\mathrm{Spa}\left(\mathbb{Z}_{p}, \mathbb{Z}_{p}\right)} \operatorname{Spa}\left(A, A^{+}\right)$. We can construct $Y$ as follows. There is an isomorphism $\mathbb{Z}_{p} \llbracket T_{1}, \ldots, T_{k} \rrbracket \cong \mathbb{Z}_{p} \llbracket \mathbb{Z}_{p}^{k} \rrbracket$ that sends $T_{i} \mapsto \Delta_{e_{i}}$, where the $e_{i}$ form a basis of $\mathbb{Z}_{p}^{k}$. For any positive rational $r=m / n$, let $B_{r}=A\left\langle T_{1}, \ldots, T_{k}, T_{1}^{n} / \alpha^{m}, \ldots, T_{k}^{n} / \alpha^{m}\right\rangle$, and let $B_{r}^{+}$be the normal closure of $A^{+}\left\langle T_{1}, \ldots, T_{n}, T_{1}^{n} / \alpha^{m}, \ldots, T_{n}^{n} / \alpha^{m}\right\rangle$ in $B_{r}$. Then $Y$ is formed by gluing the affinoids $Y_{r}:=\operatorname{Spa}\left(B_{r}, B_{r}^{+}\right)$. 
There are canonical isomorphisms

$$
\begin{aligned}
\operatorname{Hom}_{\mathbb{Z}_{p}}\left(\mathcal{C}\left(\mathbb{Z}_{p}^{k}, \mathbb{Z}_{p}\right), \mathbb{Z}_{p}\right) & \cong \mathcal{O}_{D}(D) \\
\mathcal{D}\left(\mathbb{Z}_{p}^{k}, A\right) & \cong \mathcal{O}_{Y}(Y) \\
\mathcal{D}^{(\alpha, r)}\left(\mathbb{Z}_{p}^{k}, A\right) & \cong \mathcal{O}_{Y}\left(Y_{r}\right) \quad \forall r \in \mathbb{Q}^{+} .
\end{aligned}
$$

\section{Overconvergent COHOMOLOGY}

We mostly repeat the setup of [Urb11], except that we make use of the modules defined in section 3 ,

4.1. Locally symmetric spaces. Let $\mathbb{A}\left(\right.$ resp. $\left.\mathbb{A}_{f}, \mathbb{A}_{f}^{p}\right)$ be the ring of adeles (resp. finite adeles, finite adeles away from $p$ ) of $\mathbb{Q}$.

Let $G$ be a connected reductive algebraic group over $\mathbb{Q}$. We will assume that $G\left(\mathbb{Q}_{p}\right)$ is quasisplit. Let $B, T, N, N^{-}$be compatible choices of a Borel subgroup, maximal torus, maximal unipotent subgroup, and opposite unipotent subgroup, respectively, of $G\left(\mathbb{Q}_{p}\right)$. Let $I$ be an Iwahori subgroup of $G\left(\mathbb{Q}_{p}\right)$ compatible with $B$. Then $I$ admits a factorization $I=N_{0} T_{0} N_{0}^{-}$, where $N_{0}^{-}=N^{-} \cap I, T_{0}=T \cap I, N_{0}=N \cap I$. Let $K^{p}$ be an open compact subgroup of $\mathbb{A}_{f}^{p}$, and let $K=K^{p} I$. Let $G_{\infty}^{+}$be the identity component of $G(\mathbb{R})$, and let $K_{\infty}$ be a maximal compact modulo center subgroup of $G_{\infty}^{+}$. Let $Z_{G}$ be the center of $G$.

The space

$$
\mathcal{X}:=G(\mathbb{A}) / K^{p} G_{\infty}^{+}
$$

may be considered as a locally $\mathbb{Q}_{p}$-analytic manifold. In section 3, we defined the module $\mathcal{D}_{c}(\mathcal{X}, A)$ of "locally analytic" compactly supported $A$-valued distributions on $\mathcal{X}$. 
Let $A$ be a complete Tate $\mathbb{Z}_{p}$-algebra, and let $\lambda: T_{0} \rightarrow A^{\times}$be a continuous homomorphism. By Lemma 3.3.5, $\lambda \in \mathcal{A}\left(T_{0}, A\right)$. We will assume that ker $\lambda$ contains $Z_{G}(\mathbb{Q}) K^{p} G_{\infty}^{+} \cap T_{0}$. We define $\mathcal{D}_{c, \lambda}(\mathcal{X}, A)$ to be the quotient of $\mathcal{D}_{c}(\mathcal{X}, A)$ obtained by constraining right translation by $N_{0}^{-}$to act by the identity, right translation by $T_{0}$ to act by $\lambda$, and translation by $Z_{G}(\mathbb{Q})$ to act by the identity.

The group $G(\mathbb{Q})^{\text {ad }}$ acts on $\mathcal{D}_{c, \lambda}(\mathcal{X}, A)$ by left translation. Moreover, $\mathcal{D}_{c, \lambda}(\mathcal{X}, A)$ is a direct sum of modules induced from much smaller subgroups of $G(\mathbb{Q})^{\text {ad }}$. We can write $G(\mathbb{A})$ as a finite union

$$
G(\mathbb{A})=\bigsqcup_{i} G(\mathbb{Q}) g_{i} G_{\infty}^{+} K .
$$

Let $\Gamma_{i}$ be the image of $g_{i} G_{\infty}^{+} K g_{i}^{-1} \cap G(\mathbb{Q})$ in $G(\mathbb{Q})^{\text {ad }}$. Then

$$
\mathcal{D}_{c, \lambda}(\mathcal{X}, A) \cong \bigoplus_{i} \operatorname{Ind}_{\Gamma_{i}}^{G(\mathbb{Q})^{\text {ad }}} \mathcal{D}_{\lambda}\left(g_{i} I, A\right)
$$

where $\mathcal{D}_{\lambda}\left(g_{i} I, A\right)$ is the quotient of $\mathcal{D}\left(g_{i} I, A\right)$ obtained by constraining right translation by $N_{0}^{-}$to act as the identity and right translation by $T_{0}$ to act as $\lambda$.

The existence of the Iwahori factorization implies that the map $N_{0} \rightarrow g_{i} I$ given by $n \mapsto g_{i} n$ induces an isomorphism of $A$-modules

$$
\mathcal{D}\left(N_{0}, A\right) \stackrel{\sim}{\rightarrow} \mathcal{D}_{\lambda}\left(g_{i} I, A\right) .
$$

This identification induces a $\Gamma_{i}$-action on $\mathcal{D}\left(N_{0}, A\right)$, which can be described as follows. Any $x \in I$ has an Iwahori factorization $x=\mathbf{n}(x) \mathbf{t}(x) \mathbf{n}^{-}(x)$ with $\mathbf{n}(x) \in N_{0}, \mathbf{t}(x) \in T_{0}$, $\mathbf{n}^{-}(x) \in N_{0}^{-}$, and the functions $\mathbf{n}, \mathbf{t}$, and $\mathbf{n}^{-}$are analytic. The action of $\Gamma_{i}$ on $\mathcal{D}\left(N_{0}, A\right)$ is given by

$$
\gamma \cdot[x]=\lambda\left(\mathbf{t}\left(g_{i}^{-1} \gamma g_{i} x\right)\right)\left[\mathbf{n}\left(g_{i}^{-1} \gamma g_{i} x\right)\right]
$$


for $\gamma \in \Gamma_{i}, x \in N_{0}$. Here $[x]$ denotes the Dirac delta distribution supported at $x$.

Now consider the locally symmetric space

$$
S_{G}(K):=G(\mathbb{Q}) \backslash G(\mathbb{A}) / K_{\infty} K
$$

Then $S_{G}(K) \cong \bigsqcup_{i} \mathcal{Y}_{i}$ where

$$
\mathcal{Y}_{i}:=\Gamma_{i} \backslash G_{\infty}^{+} / K_{\infty}
$$

We say that $K$ is neat if all of the $\Gamma_{i}$ are torsionfree. If that is the case, then each $\mathcal{Y}_{i}$ is a manifold with fundamental group $\Gamma_{i}$.

The manifold $S_{G}(K)$ has a Borel-Serre compactification $\overline{S_{G}(K)}$, which is homotopy equivalent to $S_{G}(K)$. Any finite triangulation of $\overline{S_{G}(K)}$ determines a resolution

$$
0 \rightarrow C_{d}\left(\Gamma_{i}\right) \rightarrow \ldots \rightarrow C_{1}\left(\Gamma_{i}\right) \rightarrow C_{0}\left(\Gamma_{i}\right) \rightarrow \mathbb{Z} \rightarrow 0
$$

where the $C_{j}\left(\Gamma_{i}\right)$ are free $\mathbb{Z}\left[\Gamma_{i}\right]$-modules of finite rank and $d$ is the dimension of $S_{G}(K)$. We define a complex $C_{\lambda}^{\bullet}$ by

$$
C_{\lambda}^{j}:=\bigoplus_{i} \operatorname{Hom}_{\Gamma_{i}}\left(C_{j}\left(\Gamma_{i}\right), \mathcal{D}_{\lambda}\left(g_{i} I, A\right)\right)
$$

Then

$$
R \Gamma^{\bullet}\left(G(\mathbb{Q})^{\text {ad }}, \mathcal{D}_{c, \lambda}(\mathcal{X}, A)\right) \cong \bigoplus_{i} R \Gamma^{\bullet}\left(\Gamma_{i}, \mathcal{D}_{\lambda}\left(g_{i} I, A\right)\right) \cong C_{\lambda}^{\bullet}
$$

in the derived category of $A$-modules.

4.2. Hecke action. We choose a projective resolution

$$
\ldots \rightarrow C_{1}\left(G(\mathbb{Q})^{\text {ad }}\right) \rightarrow C_{0}\left(G(\mathbb{Q})^{\text {ad }}\right) \rightarrow \mathbb{Z} \rightarrow 0
$$

of $\mathbb{Z}$ as a $G(\mathbb{Q})^{\text {ad }}$-module as well as maps of complexes of $\Gamma_{i}$-modules $C_{\bullet}\left(\Gamma_{i}\right) \rightarrow$ $C_{\bullet}\left(G(\mathbb{Q})^{\text {ad }}\right)$ and $C_{\bullet}\left(G(\mathbb{Q})^{\text {ad }}\right) \rightarrow C_{\bullet}\left(\Gamma_{i}\right)$ that are homotopy inverses of each other. 
Then any $f \in \operatorname{End}_{G(\mathbb{Q})^{\text {ad }}}\left(\mathcal{D}_{c, \lambda}(\mathcal{X}, A)\right)$ defines an operator $[f] \in \operatorname{End}\left(C_{\lambda}^{\bullet}\right)$ by

$$
\begin{aligned}
C_{\lambda}^{j} & \rightarrow \bigoplus_{i} \operatorname{Hom}_{\Gamma_{i}}\left(C_{j}\left(G(\mathbb{Q})^{\mathrm{ad}}\right), \mathcal{D}_{\lambda}\left(g_{i} I, A\right)\right) \\
& \stackrel{\sim}{\rightarrow} \operatorname{Hom}_{G(\mathbb{Q})^{\text {ad }}}\left(C_{j}\left(G(\mathbb{Q})^{\mathrm{ad}}\right), \mathcal{D}_{c, \lambda}(\mathcal{X}, A)\right) \\
& \stackrel{f}{\rightarrow} \operatorname{Hom}_{G(\mathbb{Q})^{\text {ad }}}\left(C_{j}\left(G(\mathbb{Q})^{\mathrm{ad}}\right), \mathcal{D}_{c, \lambda}(\mathcal{X}, A)\right) \\
& \stackrel{\sim}{\rightarrow} \bigoplus_{i} \operatorname{Hom}_{\Gamma_{i}}\left(C_{j}\left(G(\mathbb{Q})^{\mathrm{ad}}\right), \mathcal{D}_{\lambda}\left(g_{i} I, A\right)\right) \\
& \rightarrow C_{\lambda}^{j} .
\end{aligned}
$$

For any $f, g,[f][g]$ is homotopy equivalent to $[f g]$.

For any $g \in G\left(\mathbb{A}_{f}^{p}\right)$, the double coset operator $K^{p} g K^{p}$ acts on $\mathcal{D}_{c, \lambda}$ and determines a Hecke operator $\left[K^{p} g K^{p}\right]$ on $C_{\lambda}^{\bullet}$.

Let

$$
T^{-}=\left\{t \in T \mid t^{-1} N_{0}^{-} t \subseteq N_{0}^{-}\right\}
$$

For $t \in T^{-}$, the double coset operator $N_{0}^{-} t N_{0}^{-}$acts on $\mathcal{D}_{c, \lambda}$ and determines an operator $\left[N_{0}^{-} t N_{0}^{-}\right]$on $C_{\lambda}^{\bullet}$. We will sometimes denote this operator by $u_{t}$.

Our definition of the Hecke operators at $p$ differs slightly from that of previous references on overconvergent cohomology, which made use of a choice of "right *action". Our definition is instead meant to be analogous to the one used in Emerton's theory of completed cohomology [Eme06a, Eme06b]. The two approaches will yield the same eigenvariety. The only essential difference between the approaches is that, to define a "right $*$-action", one chooses a splitting of $0 \rightarrow T_{0} \rightarrow T \rightarrow T / T_{0} \rightarrow 0$, and then uses this splitting to twist the Hecke operators so that $T_{0}$ acts trivially.

Let $S$ be the set of finite places at which $K^{p}$ is not maximal hyperspecial. Let $\mathbb{A}_{f}^{p, S}$ be the adeles away from $p$ and $S$, and let $K^{p, S}$ be the image of $K^{p}$ in $\mathbb{A}_{f}^{p, S}$. We define 
the Hecke algebra

$$
\mathcal{H}_{G}:=C_{c}^{\infty}\left(K^{p, S} \backslash G\left(\mathbb{A}_{f}^{p, S}\right) / K^{p, S} \times N_{0}^{-} \backslash N_{0}^{-} T^{-} N_{0}^{-} / N_{0}^{-}, \mathbb{Z}_{p}\right)
$$

4.3. Topological properties of Hecke operators. In order to apply the spectral theory introduced in section 2.2 , we will need to choose a particular description of $C_{\lambda}^{\bullet}$ as a limit of complexes of projective modules. We define an abelian group structure on $N_{0}$ by $\left(n, n^{\prime}\right) \mapsto \exp \left(\log (n)+\log \left(n^{\prime}\right)\right)$. This structure allows us to define the projective modules $\mathcal{D}^{(\alpha, r)}\left(N_{0}, A\right)$ for some arbitrarily chosen topologically nilpotent unit $\alpha \in A$. We define

$$
C_{\lambda, \alpha, r}^{i}:=\bigoplus_{j} \operatorname{Hom}_{\Gamma_{j}}\left(C_{i}\left(\Gamma_{j}\right), \mathcal{D}^{(\alpha, r)}\left(N_{0}, A\right)\right)
$$

Lemma 4.3.1. For all sufficiently small $r$ and all $\epsilon>0$, the differential $d: C_{\lambda}^{i+1} \rightarrow$ $C_{\lambda}^{i}$ extends to a map $C_{\lambda, \alpha, r}^{i+1} \rightarrow C_{\lambda, \alpha, r+\epsilon}^{i}$.

Proof. It is enough to check that for sufficiently small $r$ and all $\epsilon>0$, left translation by any $\gamma \in \Gamma_{i}$ maps $\mathcal{D}^{(\alpha, r)}\left(N_{0}, A\right)$ into $\mathcal{D}^{(\alpha, r+\epsilon)}\left(N_{0}, A\right)$. This follows from the description of the action in section 4.1 along with Lemmas 3.3.1 and 3.3.5 and Proposition 3.3 .3 .

If $\underline{r}=\left(r_{0}, \ldots, r_{d}\right)$ is chosen such that the differentials $C_{\lambda, \alpha, r_{i+1}}^{i+1} \rightarrow C_{\lambda, \alpha, r_{i}}^{i}$ are defined, then we denote the corresponding complex by $C_{\lambda, \alpha, \underline{r}}^{\bullet}$.

Choose some $t \in T^{-}$such that $t^{-1} N_{0} t \subset N_{0}^{p}$. Let $\mathcal{H}_{G}^{\prime}$ be the ideal of $\mathcal{H}_{G}$ generated by $u_{t}$.

Lemma 4.3.2. There exists $r_{0} \in \mathbb{R}^{+}$so that for all $r \in\left(0, r_{0}\right), \in \in \mathbb{R}^{+}$, and $f \in$ $\mathcal{H}_{G}^{\prime}, f$ determines a continuous map $C_{\lambda, \alpha, r}^{i} \rightarrow C_{\lambda, \alpha, r / p+\epsilon}^{i}$, and hence $f$ determines a completely continuous map $C_{\lambda, \alpha, r}^{i} \rightarrow C_{\lambda, \alpha, r}^{i}$. 
Proof. We can show that Hecke operators away from $p$ map $C_{\lambda, \alpha, r}^{i}$ into $C_{\lambda, \alpha, r+\epsilon}^{i}$ using essentially the same argument as in Lemma 4.3.1. It remains to show that $u_{t}$ maps $C_{\lambda, \alpha, r}^{i}$ into $C_{\lambda, \alpha, r / p+\epsilon}^{i}$. The action of $u_{t}$ can be built from functions of the form

$$
[x] \mapsto \lambda(\mathbf{t}(\iota(x)))[\mathbf{n}(\iota(x))]
$$

where $\iota(x)$ takes the form

$$
\iota(x)=h t^{-1} \mathbf{n}\left(n^{-} x\right) \mathbf{t}\left(n^{-} x\right) t
$$

for some $n^{-} \in N_{0}^{-}, h \in I$. (See for example Eme06a, Lemma 4.2.19].) In particular, $\mathbf{n}(\iota(x))$ belongs to a single right coset of $t^{-1} N_{0} t \subset N_{0}^{p}$. The argument proceeds as before, except that we also need to use Proposition 3.3.4 and Lemma 3.3.6.

4.4. Characteristic power series. For any $f \in \mathcal{H}_{G}^{\prime}$ we define the power series

$$
\operatorname{det}\left(1-X f \mid C_{\lambda}^{\bullet}\right):=\operatorname{det}\left(1-X f \mid C_{\lambda, \alpha, \underline{r}}^{\bullet}\right)
$$

for any $\alpha, \underline{r}$ for which the complex $C_{\lambda, \alpha, \underline{r}}^{\bullet}$ is defined and the $u_{t}$ operator is completely continuous. Choosing a different $\alpha$ and $\underline{r}$ conjugates the matrix of $f$ by a diagonal matrix, so the power series does not depend on them.

Similarly, we define $\operatorname{det}\left(1-X f \mid C_{\lambda}^{i}\right)$ to be $\operatorname{det}\left(1-X f \mid C_{\lambda, \alpha, \underline{r}}^{i}\right)$. Consider the Fredholm series

$$
P_{+}(X)=\prod_{i=0}^{d} \operatorname{det}\left(1-X u_{t} \mid C_{\lambda}^{i}\right) .
$$

Suppose that $P_{+}(X)$ factors as $Q_{+}(X) S_{+}(X)$, with $Q_{+}(X) \in A[X], S_{+}(X) \in A\{\{X\}\}$, that $Q_{+}(X)$ and $S_{+}(X)$ are relatively prime, and that the leading coefficient of $Q_{+}(X)$ 
is invertible. Let $Q_{+}^{*}(X)=X^{\operatorname{deg} Q_{+}} Q_{+}\left(X^{-1}\right)$. By AIP, Théorème B.2], there is a decomposition $C_{\lambda, \alpha, \underline{r}}^{\bullet}=N_{\alpha, \underline{r}}^{\bullet} \oplus F_{\alpha, \underline{r}}^{\bullet}$, where $Q_{+}^{*}\left(u_{t}\right)$ annihilates $N_{\alpha, \underline{r}}^{\bullet}$ and acts invertibly on $F_{\alpha, \underline{r}}^{\bullet}$, and the $N_{\alpha, \underline{r}}^{i}$ are finitely generated and projective.

Lemma 4.4.1. For any $\alpha, \alpha^{\prime}$ and $\underline{r}, \underline{r}^{\prime}$ such that $N_{\alpha, \underline{\underline{r}}}^{\bullet}$ and $N_{\alpha^{\prime}, \underline{r}^{\prime}}^{\bullet}$ are defined, they are canonically isomorphic.

Proof. Choose $\underline{r}^{\prime \prime}$ so that $C_{\lambda, \alpha, \underline{r}^{\prime \prime}}$ injects into $C_{\lambda, \alpha, \underline{\underline{r}}}^{\bullet}$ and $C_{\lambda, \alpha^{\prime}, \underline{r}^{\prime}}$. The operator $1-\frac{Q_{+}^{*}\left(u_{t}\right)}{Q_{+}^{*}(0)}$ acts as the identity on $N_{\alpha, \underline{r}}^{\bullet}$, and for sufficiently large $n,\left(1-\frac{Q_{+}^{*}\left(u_{t}\right)}{Q_{+}^{*}(0)}\right)^{n}$ factors through $N_{\alpha, \underline{r}^{\prime \prime}}^{\bullet}$. So we get a canonical isomorphism $N_{\alpha, \underline{\underline{r}}}^{\bullet} \cong N_{\alpha, \underline{r}^{\prime \prime}}^{\bullet}$, and similarly there is a canonical isomorphism $N_{\alpha^{\prime}, r^{\prime}}^{\bullet} \cong N_{\alpha, r^{\prime \prime}}^{\bullet}$.

Corollary 4.4.2. There is a decomposition $C_{\lambda}^{\bullet}=N^{\bullet} \oplus F^{\bullet}$, where $Q_{+}^{*}\left(u_{t}\right)$ annihilates $N^{\bullet}$ and acts invertibly on $F^{\bullet}$, and the $N^{i}$ are finitely generated and projective.

\section{EisEnStein AND CUSPIDAl CONTRIBUtions TO CHARACTERISTIC POWER} SERIES

5.1. Preliminaries. In this section, we will write $C_{G, K^{p}, \lambda}^{\bullet}$ for $C_{\lambda}^{\bullet}$ to make it clear which group we are considering. We will also assume that $G(\mathbb{R})$ has discrete series, since otherwise Urban's eigenvariety will be empty.

In order to construct Urban's eigenvariety, we need the characteristic power series of the Hecke operators to be Fredholm series. However, the power series $\operatorname{det}\left(1-X f \mid C_{G, K^{p}, \lambda}^{\bullet}\right)$ includes contributions from both cusp forms and Eisenstein series, and the Eisenstein contribution is generally only a ratio of Fredholm series. We will now define a complex $C_{G, K^{p}, \lambda, \text { cusp }}$ whose characteristic power series only includes contributions from cusp forms. (This complex will only be useful for defining characteristic power series; we make no attempt to remove the Eisenstein series from the cohomology.) 
We will mostly follow [Urb11, §4.6]. However, there is an error in the handling of the Eisenstein series in [Urb11] that we will need to correct. The region of convergence of an Eisenstein series is generally not a union of Weyl chambers. (For example, $S p(6)$ has two conjugacy classes of parabolic subgroups whose Levis are isomorphic to $G L(2) \times G L(1)$. The region of convergence of Eisenstein series coming from these parabolics contains one or two full Weyl chambers and fractions of three others.) Consequently, the set $\mathcal{W}_{\text {Eis }}^{M}$ defined in [Urb11 should depend on the weight of the Eisenstein series. A more careful argument is therefore needed to show that character distribution $I_{G, 0}^{c l}(f, \mu)$ has a unique $p$-adic interpolation. In fact, it appears that the character distribution of Eisenstein series coming from a single parabolic subgroup will generally not have a unique interpolation. We will show, however, that the sum of distributions coming from parabolic subgroups that have a common Levi will have a unique interpolation.

We let $W_{G}$ denote the Weyl group of $G$. We let $\Phi_{G}, \Phi_{G}^{\vee}$ denote the set of roots and coroots, respectively, of the pair $\left(G_{\mathbb{Q}_{p}}, T\right)$, where $T$ is the torus chosen in section 4. We let $\Phi_{G}^{+}$(resp. $\Phi_{G}^{-}$) denote the subset of roots that are positive (resp. negative) with respect to $B$, and we make a similar definition for coroots. We let $\rho$ denote half the sum of the positive roots.

Let $F$ be a finite extension of $\mathbb{Q}_{p}$. We say that $\mu: T_{0} \rightarrow F^{\times}$is an algebraic weight if it can be extended to a homomorphism of algebraic groups $T_{F} \rightarrow\left(\mathbb{G}_{m}\right)_{F}$. We say that an algebraic weight $\mu$ is dominant (resp. regular dominant) if $\left\langle\alpha^{\vee}, \mu\right\rangle \geq 0$ (resp. >0) for all $\alpha^{\vee} \in \Phi_{G}^{\vee+}$.

Suppose that $\mu$ is dominant. Then $\mathcal{D}_{\mu}\left(g_{i} I, F\right)$ has a (nonzero) quotient that is a finite-dimensional $F$-vector space. We will write $L_{\mu}^{G}$ for the corresponding local system on either $S_{G}(K)$ or $\overline{S_{G}(K)}$. 
Lemma 5.1.1. Let $f=u_{t} \otimes f^{p} \in \mathcal{H}_{G}^{\prime}$, and let $\mu: T_{0} \rightarrow F^{\times}$be an algebraic dominant weight. Then

$$
\operatorname{det}\left(1-X f \mid C_{G, K^{p}, \mu}^{\bullet}\right) \equiv \operatorname{det}\left(1-X f \mid H^{\bullet}\left(S_{G}(K), L_{\mu}^{G}\right)\right) \quad\left(\bmod \mathcal{O}_{F} \llbracket N(\mu, t) X \rrbracket\right)
$$

where

$$
N(\mu, t)=\inf _{w \in W_{G} \backslash\{\operatorname{id}\}}\left|t^{(w-1)(\mu+\rho)}\right|_{p}
$$

Proof. For the degree 1 term, this is [Urb11, Lemma 4.5.2]. The argument used there also works for higher degree terms.

In section 7, we will consider a family of weights having the property that for any $n \in \mathbb{N}$, the set of points corresponding to regular dominant weights $\mu$ satisfying $p^{n} \mid N(\mu, t)$ is Zariski dense. The characteristic power series for the whole family can then be determined from the $\operatorname{det}\left(1-X f \mid H^{\bullet}\left(S_{G}(K), L_{\mu}^{G}\right)\right)$.

If $\mu$ is regular dominant, then the cuspidal subspace of $H^{i}\left(S_{G}(K), L_{\mu}^{G}\right)$ is the interior cohomology $H_{!}^{i}\left(S_{G}(K), L_{\mu}^{G}\right)[\mathrm{LS} 04, \S 5.3]$, and furthermore (since we assume $G(\mathbb{R})$ has discrete series) the interior cohomology is nonzero only in the middle degree BW00, Theorem III.5.1]. Hence either $\operatorname{det}\left(1-X f \mid H_{!}^{\bullet}\left(S_{G}(K), L_{\mu}^{G}\right)\right)$ or its reciprocal is a polynomial.

Our goal is to prove a version of Lemma 5.1.1 in which $C_{G, K^{p}, \mu}^{\bullet}$ is replaced by a complex $C_{G, K^{p}, \mu, \text { cusp }}^{\bullet}$ that we will define, and $H^{\bullet}\left(S_{G}(K), L_{\mu}^{G}\right)$ is replaced by $H_{!}^{\bullet}\left(S_{G}(K), L_{\mu}^{G}\right)$. 5.2. Cohomology of the Borel-Serre boundary. Eisenstein series arise from the Borel-Serre boundary $\partial S_{G}(K):=\overline{S_{G}(K)} \backslash S_{G}(K)$ of $S_{G}(K)$. The boundary has a stratification by locally symmetric spaces of parabolic subgroups of $G$.

We warn the reader that the Borel-Serre compactification of $\overline{S_{G}(K)}$ slightly strange. When constructing a locally symmetric space, one usually takes a quotient by the identity component of either $Z_{G}(\mathbb{R})$ or $A_{G}(\mathbb{R})$, where $A_{G}$ is the $\mathbb{Q}$-split part of $Z_{G}$. In 
order to construct Urban's eigenvariety, we need to choose the former option, but the Borel-Serre compactification behaves better with respect to the latter. Consequently, if $M$ is a Levi subgroup of $G$, then the locally symmetric space for $M$ should be constructed by taking a quotient by the identity component of $Z_{G}(\mathbb{R}) A_{M}(\mathbb{R})$ rather than that of $Z_{M}(\mathbb{R})$. However, it will turn out that we only need to consider Levi subgroups for which the two quotients are the same; see section 5.4 for more details.

Let $P$ be a parabolic subgroup of $G$, let $N$ be the maximal unipotent subgroup of $P$, and let $M=P / N$ be its Levi quotient. Let $K_{P}^{p}=K^{p} \cap P\left(\mathbb{A}_{f}^{p}\right), K_{P, p}=I \cap P\left(\mathbb{Q}_{p}\right)$, $K_{P}=K_{P}^{p} K_{P, p}$. We can define a locally symmetric space $S_{P}\left(K_{P}\right)$, and there is a locally closed immersion

$$
\iota: S_{P}\left(K_{P}\right) \rightarrow \overline{S_{G}(K)} .
$$

If $P^{\prime}$ is another parabolic subgroup of $G$, then $S_{P}\left(K_{P}\right)$ and $S_{P^{\prime}}\left(K_{P^{\prime}}\right)$ will have the same image in $\overline{S_{G}(K)}$ if and only if $P\left(\mathbb{A}_{f}\right)$ and $P^{\prime}\left(\mathbb{A}_{f}\right)$ are conjugate by an element of $K^{p} I$.

Let $K_{M}^{p}, I_{M}$ be the images of $K_{P}^{p}, K_{P, p}$ in $M\left(\mathbb{A}_{f}^{p}\right), M\left(\mathbb{Q}_{p}\right)$, respectively. The group $I_{M}$ is an Iwahori subgroup of $M$. Let $K_{M}=I_{M} K_{M}^{p}$. The locally symmetric space $S_{P}\left(K_{P}\right)$ is a nilmanifold bundle over $S_{M}\left(K_{M}\right)$. We let

$$
\pi: S_{P}\left(K_{P}\right) \rightarrow S_{M}\left(K_{M}\right)
$$

denote the projection.

We can relate $R \pi_{*} \iota^{*} L_{\mu}^{G}$ to local systems on $S_{M}\left(K_{M}\right)$ using the Kostant decomposition BW00, Theorem III.3.1]. To define the local systems on $S_{M}\left(K_{M}\right)$, we first need to choose a quasisplit torus $T_{M}$ of $M$. Since $G\left(\mathbb{Q}_{p}\right)=P\left(\mathbb{Q}_{p}\right) I$, we can choose $i \in I$ so that $i T i^{-1} \subset P_{\mathbb{Q}_{p}}$. We let $T_{M}$ be the image of $i T i^{-1}$ in $M$. We let $w \in W_{G}$ be the minimal length element satisfying $i w\left(B_{\overline{\mathbb{Q}}_{p}}\right) w^{-1} i^{-1} \subset P_{\overline{\mathbb{Q}}_{p}}$. The isomorphism 
$T \stackrel{\sim}{\rightarrow} T_{M}$ defined by $t \mapsto i w t w^{-1} i^{-1}$ determines a length-preserving injection of Weyl groups $W_{M} \hookrightarrow W_{G}$. We let $W^{M}$ denote a set of minimal length coset representatives of $W_{M} \backslash W_{G}$.

We have the following isomorphism in the derived category of constructible sheaves on $S_{M}\left(K_{M}\right)$.

$$
R \pi_{*} \iota^{*} L_{\mu}^{G} \cong \bigoplus_{w^{\prime} \in W^{M}} L_{w^{-1}\left(w^{\prime}(\mu+\rho)-\rho\right)}^{M}\left[l\left(w^{\prime}\right)-\operatorname{dim} N\right]
$$

Here $l\left(w^{\prime}\right)$ denotes the length of $w^{\prime}$. To see that the splitting exists in the derived category and not just at the level of cohomology, we observe that the $L_{w^{-1}\left(w^{\prime}(\mu+\rho)-\rho\right)}^{M}$ have distinct central characters.

5.3. Hecke action. We will now define an action of $\mathcal{H}_{G}$ on the cohomology of $S_{M}\left(K_{M}\right)$ by constructing a homomorphism $\mathcal{H}_{G} \rightarrow \mathcal{H}_{M}$. The map $R \pi_{*} \iota^{*}$ will be equivariant for this action.

As explained in [Urb11, Corollary 4.6.3], for any summand of $(5.2 .1)$ with $w \neq w^{\prime}$, the Hecke eigenvalues of $u_{t} \in \mathcal{H}_{G}^{\prime}$ acting on the cohomology of this summand will be divisible by $N(\mu, t)$. We therefore only need to consider the summand with $w=w^{\prime}$. (In fact we should ignore the other summands, because the corresponding Eisenstein series are $p$-adic limits of cusp forms.) We are therefore only interested in the local system

$$
L_{w^{-1}(w(\mu+\rho)-\rho)}^{M}=L_{\mu+\left(1-w^{-1}\right) \rho}^{M} .
$$

Our definition of the homomorphism $\mathcal{H}_{G} \rightarrow \mathcal{H}_{M}$ will be the same as that of Urb11, 4.1.8], except that our convention for the Hecke operators makes some normalization factors disappear. The Hecke algebra $\mathcal{H}_{G}$ is generated by operators of the form $u_{t}$ for $t \in T^{-}$and $\left[K_{v} g K_{v}\right]$ for $v \notin S, g \in G\left(\mathbb{Q}_{v}\right)$. We let $u_{t} \in \mathcal{H}_{G}$ act as $t^{\left(1-w^{-1}\right) \rho} u_{t} \in \mathcal{H}_{M}$. 
The double coset $K_{v} g K_{v}$ decomposes as a finite union $\bigsqcup_{j} K_{v} p_{j} K_{v}$ with $p_{j} \in P\left(\mathbb{Q}_{v}\right)$. We let $\left[K_{v} g K_{v}\right]$ act as $\sum_{j}\left[K_{M, v} m_{j} K_{M, v}\right]$, where $m_{j}$ is the image of $p_{j}$ in $M\left(\mathbb{Q}_{v}\right)$.

Lemma 5.3.1. The homomorphism $\mathcal{H}_{G} \rightarrow \mathcal{H}_{M}$ defined above makes the map

$$
R \pi_{*} \iota^{*}: H^{\bullet}\left(S_{G}(K), L_{\mu}^{G}\right) \rightarrow H^{\bullet}\left(S_{M}\left(K_{M}\right), L_{\mu+\left(1-w^{-1}\right) \rho}^{M}\right)[l(w)-\operatorname{dim} N]
$$

$\mathcal{H}_{G^{-}}$equivariant.

Proof. The argument is essentially the same as that of [Urb11, 4.1.8, 4.6.1-3].

5.4. Image of the map $R \pi_{*} \iota^{*}$. To simplify some of the analysis that follows, we will observe that some Levis have $H^{\bullet}\left(S_{M}\left(K_{M}\right), L_{\mu}^{M}\right)=0$ for a Zariski dense subset of weights $\mu$, and hence they cannot contribute to the characteristic power series. The Levi $M$ can have a nonzero contribution only if the following conditions hold (see [Urb11, Theorem 4.7.3(ii)']):

(1) $M(\mathbb{R})$ has discrete series.

(2) The center $Z_{M}$ of $M$ is generated by its maximal split subgroup, its maximal compact subgroup, and $Z_{G}$.

Let $T_{M}^{\prime}$ be a maximally compact maximal torus of $M$. Any choice of embed$\operatorname{ding} \overline{\mathbb{Q}} \rightarrow \mathbb{C}$ determines an action of complex conjugation on the cocharacter lattice $X_{*}\left(T_{M}^{\prime} / Z_{G}\right)$. Choosing an identification of $X_{*}\left(T_{M} / Z_{G}\right)$ with $X_{*}\left(T_{M}^{\prime} / Z_{G}\right)$ gives an involution

$$
\theta: X_{*}\left(T_{M} / Z_{G}\right) \rightarrow X_{*}\left(T_{M} / Z_{G}\right)
$$

The two assumptions listed above guarantee that $\theta$ is actually independent of all choices. More specifically, the first assumption guarantees that $T_{M}^{\prime} / Z_{M}$ is compact and therefore the induced map $X_{*}\left(T_{M}^{\prime} / Z_{M}\right) \rightarrow X_{*}\left(T_{M}^{\prime} / Z_{M}\right)$ is minus the identity. The 
second assumption guarantees that the induced map $X_{*}\left(Z_{M} / Z_{G}\right) \rightarrow X_{*}\left(Z_{M} / Z_{G}\right)$ is independent of the embedding $\overline{\mathbb{Q}} \rightarrow \mathbb{C}$.

By [LS04, §3.2], the image of

$$
R \pi_{*} \iota^{*}: H^{\bullet}\left(S_{G}(K), L_{\mu}^{G}\right) \rightarrow H^{\bullet}\left(S_{M}\left(K_{M}\right), L_{\mu+\left(1-w^{-1}\right) \rho}^{M}\right)[l(w)-\operatorname{dim} N]
$$

can have nonzero intersection with the cuspidal part

$$
H_{!}^{\bullet}\left(S_{M}\left(K_{M}\right), L_{\mu+\left(1-w^{-1}\right) \rho}^{M}\right)[l(w)-\operatorname{dim} N]
$$

only if

$$
\left.\left\langle\alpha^{\vee}, w(1+\theta)(\mu+\rho)\right)\right\rangle<0 \quad \forall \alpha^{\vee} \in \Phi_{G}^{\vee+} \backslash \Phi_{M}^{\vee+}
$$

If the above equation holds and no Eisenstein series arising from $M$ has a pole at $-w(\mu+\rho)$, then the image contains the cuspidal part. In particular, the image contains the cuspidal part if $(5.4 .1)$ is satisfied and

$$
\left|\left\langle\alpha^{\vee},(1+\theta) \mu\right\rangle\right| \geq 4\left|\left\langle\alpha^{\vee}, \rho\right\rangle\right| \quad \forall \alpha^{\vee} \in \Phi_{G}^{\vee} \backslash \Phi_{M}^{\vee}
$$

The constraint (5.4.1) is archimedean in nature, and therefore appears to provide an obstacle to interpolating Eisenstein series $p$-adically. To get around this issue, we will combine contributions from parabolic subgroups having common Levis.

We will call a Levi subgroup "relevant" if it satisfies the two conditions listed at the beginning of this section, and we will call a parabolic subgroup relevant if its Levi is relevant. Let $\mathcal{P}_{0}$ be the set of relevant parabolic subgroups of $G$, modulo the relation that $P_{1}$ and $P_{2}$ are considered equivalent if $P_{1}\left(\mathbb{A}_{f}\right)$ and $P_{2}\left(\mathbb{A}_{f}\right)$ are conjugate by an element of $K^{p} I$. Let $\mathcal{P}$ be the set of relevant parabolic subgroups of $G$, modulo the relation that $P_{1}$ and $P_{2}$ are considered equivalent if $P_{1}\left(\mathbb{Q}_{p}\right)$ and $P_{2}\left(\mathbb{Q}_{p}\right)$ are conjugate 
by an element of $I$. Let $\mathcal{M}$ be the set of relevant Levi subgroups of $G$, modulo the relation that $M_{1}$ and $M_{2}$ are considered equivalent if $M_{1}\left(\mathbb{Q}_{p}\right)$ and $M_{2}\left(\mathbb{Q}_{p}\right)$ are conjugate by an element of $I$. There are surjections $\mathcal{P}_{0} \rightarrow \mathcal{P} \rightarrow \mathcal{M}$.

Choose representatives of each element of $\mathcal{P}_{0}$ and $\mathcal{M}$. If $P$ is the representative of an element of $\mathcal{P}_{0}$ and $M$ is the representative of its image in $\mathcal{M}$, choose a $g \in G(\mathbb{Q})$ so that $M \subset g P g^{-1}$ and the image of $g$ in $G\left(\mathbb{Q}_{p}\right)$ is in $I$. This choice determines an identification of $M$ with the Levi quotient of $P$. We will sometimes identify elements of $\mathcal{P}_{0}$ and $\mathcal{M}$ with the chosen representatives.

Let $M \in \mathcal{M}$. Assume $\mu$ is chosen so that (5.4.2) is satisfied. Then there is exactly one parabolic subgroup $P_{\mu}$ containing $M$ for which (5.4.1) will be satisfied: it is the parabolic determined by the set of coroots $\alpha^{\vee}$ satisfying

$$
\left\langle\alpha^{\vee},(1+\theta) \mu\right\rangle<0 .
$$

So $\mu$ determines a section $\mathcal{M} \rightarrow \mathcal{P}$ of the projection $\mathcal{P} \rightarrow \mathcal{M}$. Let $\mathcal{P}_{\mu}$ be the image of this section, and let $\mathcal{P}_{0, \mu}$ be the preimage of $\mathcal{P}_{\mu}$ in $\mathcal{P}_{0}$. At the end of section 5.2 , we associated each parabolic subgroup of $G$ with an element of $W_{G}$; this association determines a map $w: \mathcal{P} \rightarrow W_{G}$.

\section{Lemma 5.4.3.}

$$
\begin{gathered}
l\left(w\left(P_{\mu}\right)\right)=\frac{1}{2}\left|\left(\Phi_{G}^{-} \backslash \Phi_{M}^{-}\right) \cap \theta\left(\Phi_{G}^{+} \backslash \Phi_{M}^{+}\right)\right| \\
(1-\theta)\left(1-w\left(P_{\mu}\right)^{-1}\right) \rho=\sum_{\alpha \in\left(\Phi_{G}^{-} \backslash \Phi_{M}^{-}\right) \cap \theta\left(\Phi_{G}^{+} \backslash \Phi_{M}^{+}\right)} \alpha
\end{gathered}
$$

In particular, $l\left(w\left(P_{\mu}\right)\right)$ and $(1-\theta)\left(1-w\left(P_{\mu}\right)^{-1}\right) \rho$ do not depend on $\mu$.

Proof. By definition,

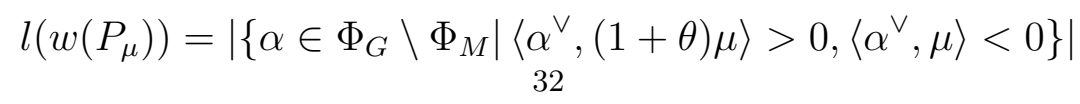


Observe that if $\left\langle\alpha^{\vee}, \mu\right\rangle<0<\left\langle\alpha^{\vee}, \theta \mu\right\rangle$, then exactly one of the inequalities

$$
\left\langle\alpha^{\vee}, \mu\right\rangle<0<\left\langle\alpha^{\vee},(1+\theta) \mu\right\rangle, \quad\left\langle\left(-\alpha^{\vee} \theta\right), \mu\right\rangle<0<\left\langle\left(-\alpha^{\vee} \theta\right),(1+\theta) \mu\right\rangle
$$

will be satisfied, and otherwise neither will be satisfied. This observation also proves the second item.

We will write $l(M)$ for $l\left(w\left(P_{\mu}\right)\right)$ and $\rho(M, \mu)$ for $\left(1-w\left(P_{\mu}\right)^{-1}\right) \rho$.

Now we are almost ready to write down an analogue of Lemma 5.1.1 for cusp forms. The boundary components of $S_{G}(K)$ whose Eisenstein series contribute to the characteristic power series $\operatorname{det}\left(1-X f \mid H^{\bullet}\left(S_{G}(K), L_{\mu}^{G}\right)\right) \bmod \mathcal{O}_{F} \llbracket N(\mu, t) X \rrbracket$ are in bijection with elements of $\mathcal{P}_{0, \mu}$. Given $M \in \mathcal{M}$, a choice of a preimage $P$ of $M$ in $\mathcal{P}_{0, \mu}$ determines an open compact subgroup $K_{M}^{p}$ of $M\left(\mathbb{A}_{f}^{p}\right)$, as described in section 5.2 . Let $\mathcal{K}_{M}^{p}$ be the collection of all such subgroups.

The analysis of the last few sections gives us the following identity.

Lemma 5.4.4. For any dominant algebraic weight $\mu: T \rightarrow F^{\times}$satisfying (5.4.2),

$$
\begin{aligned}
& \frac{\operatorname{det}\left(1-X f \mid H^{\bullet}\left(S_{G}(K), L_{\mu}^{G}\right)\right)}{\operatorname{det}\left(1-X f \mid H_{!}^{\bullet}\left(S_{G}(K), L_{\mu}^{G}\right)\right)} \\
\equiv & \prod_{M \in \mathcal{M}} \prod_{K_{M} \in \mathcal{K}_{M, \mu}^{p}} \operatorname{det}\left(1-X f \mid H_{!}^{\bullet}\left(S_{M}\left(K_{M}\right), L_{\mu+\rho(M, \mu)}^{M}\right)\right)^{(-1)^{\operatorname{dim} N-l(M)}} \quad\left(\bmod \mathcal{O}_{F} \llbracket N(\mu, t) X \rrbracket\right)
\end{aligned}
$$

In order to interpolate the local systems $p$-adically, we need something slightly more general. 
Proposition 5.4.5. For any dominant algebraic weights $\mu: T \rightarrow F^{\times}$and $\mu_{0}: T \rightarrow$ $F_{0}^{\times}$satisfying (5.4.2),

$$
\begin{aligned}
& \frac{\operatorname{det}\left(1-X f \mid H^{\bullet}\left(S_{G}(K), L_{\mu}^{G}\right)\right)}{\operatorname{det}\left(1-X f \mid H_{!}^{\bullet}\left(S_{G}(K), L_{\mu}^{G}\right)\right)} \\
\equiv & \prod_{M \in \mathcal{M}} \prod_{K_{M} \in \mathcal{K}_{M, \mu_{0}}} \operatorname{det}\left(1-X f \mid H_{!}^{\bullet}\left(S_{M}\left(K_{M}\right), L_{\mu+\rho\left(M, \mu_{0}\right)}^{M}\right)\right)^{(-1)^{\operatorname{dim} N-l(M)}} \quad\left(\bmod \mathcal{O}_{F} \llbracket N(\mu, t) X \rrbracket\right)
\end{aligned}
$$

Proof. We claim that local systems $L_{\mu+\rho(M, \mu)}^{M}, L_{\mu+\rho\left(M, \mu_{0}\right)}^{M}$ are isomorphic. The isomorphism class of each local system depends only the restriction of the weight to $M^{\text {der }}$. The operator $\frac{1-\theta}{2}$ acts as the identity on the character lattice of $M^{\text {der }}$, so the claim follows from Lemma 5.4.3. Furthermore, the isomorphism of local systems induces an $\mathcal{H}_{G}$-equivariant isomorphism on cohomology. (The isomorphism on cohomology is not $\mathcal{H}_{M}$-equivariant- the actions of $u_{t}$ differ by a factor of $t^{\rho(M, \mu)-\rho\left(M, \mu_{0}\right)}$. However, the two homomorphisms $\mathcal{H}_{G} \rightarrow \mathcal{H}_{M}$ also differ by the same factor, and so the differences cancel each other.)

It remains to explain why can replace $\mathcal{K}_{M, \mu}$ with $\mathcal{K}_{M, \mu_{0}}$. Essentially, we need to show that if $\pi=\pi_{\infty} \otimes \pi_{p} \otimes \pi_{f}^{p}$ is an automorphic representation of $M$, then

$$
\sum_{K_{M}^{p} \in \mathcal{K}_{M, \mu}^{p}} \operatorname{tr}\left(\mathbf{1}_{K_{M}^{p}} \mid \pi_{f}^{p}\right)=\operatorname{tr}\left(\mathbf{1}_{K^{p}} \mid \operatorname{Ind}_{P_{\mu}\left(\mathbb{A}_{f}^{p}\right)}^{G\left(\mathbb{A}_{f}^{p}\right)} \pi_{f}^{p}\right)
$$

is independent of $\mu$. By [BZ77, 2.9-2.10], for any place $v$, the composition series of the local factor of $\operatorname{Ind}_{P_{\mu}\left(\mathbb{A}_{f}\right)}^{G\left(\mathbb{A}_{f}\right)} \pi_{f}^{p}$ at $v$ is independent of $\mu$. It follows that the trace of $\mathbf{1}_{K^{p}}$ does not depend on $\mu$.

5.5. The complex $C_{G, K^{p}, \lambda, \text { cusp }}^{\bullet}$. Now we fix an algebraic dominant weight $\mu_{0}$, and let $\lambda: T \rightarrow A^{\times}$be any weight. We define $C_{G, K^{p}, \lambda, \text { cusp }}^{\bullet}$ inductively, assuming that 
analogous complexes have already been defined for $M \in \mathcal{M}$.

$$
C_{G, K^{p}, \lambda, \text { cusp }}^{\bullet}:=C_{G, K^{p}, \lambda}^{\bullet} \oplus \bigoplus_{M \in \mathcal{M}} \bigoplus_{K_{M}^{p} \in \mathcal{K}_{M, \mu_{0}}^{p}} C_{M, K_{M}^{p}, \lambda+\rho\left(M, \mu_{0}\right), \operatorname{cusp}}^{\bullet}[l(M)-\operatorname{dim} N-1]
$$

Proposition 5.5.1. Let $F$ be a finite extension of $\mathbb{Q}_{p}$, let $\mu: T \rightarrow F^{\times}$be an algebraic dominant weight, and let $f=u_{t} \otimes f^{p} \in \mathcal{H}_{G}^{\prime}$. If $\mu$ is sufficiently general, then

$$
\operatorname{det}\left(1-X f \mid C_{G, K^{p}, \mu, \text { cusp }}^{\bullet}\right) \equiv \operatorname{det}\left(1-X f \mid H_{!}^{\bullet}\left(S_{G}(K), L_{\mu}^{G}\right)\right) \quad\left(\bmod \mathcal{O}_{F} \llbracket N(\mu, t) X \rrbracket\right) .
$$

Proof. By induction, we may assume that the proposition holds for all Levi subgroups of $G$.

$$
\begin{aligned}
& \operatorname{det}\left(1-X f \mid C_{G, K^{p}, \mu, \text { cusp }}^{\bullet}\right) \\
\equiv & \operatorname{det}\left(1-X f \mid H^{\bullet}\left(S_{G}(K), L_{\mu}^{G}\right)\right) \prod_{M, K_{M}} \operatorname{det}\left(1-X f \mid H_{!}^{\bullet}\left(S_{M}\left(K_{M}\right), L_{\mu+\rho\left(M, \mu_{0}\right)}^{M}\right)\right)^{(-1)^{l(M)-\operatorname{dim} N+1}} \\
\equiv & \operatorname{det}\left(1-X f \mid H_{!}^{\bullet}\left(S_{G}(K), L_{\mu}^{G}\right)\right) \quad\left(\bmod \mathcal{O}_{F} \llbracket N(\mu, t) X \rrbracket\right)
\end{aligned}
$$

where we used the induction hypothesis and Lemma 5.1.1 in the second line and Proposition 5.4.5 in the third line. We also use the fact that $\rho\left(M, \mu_{0}\right)$ is $M$-dominant, and so $\mathcal{O}_{F} \llbracket N\left(\mu+\rho\left(M, \mu_{0}\right), t\right) X \rrbracket \subseteq \mathcal{O}_{F} \llbracket N(\mu, t) X \rrbracket$.

The analysis of section 4.4 applies equally well to $C_{G, K^{p}, \lambda, \text { cusp }}^{\bullet}$ For any $f \in \mathcal{H}_{G}^{\prime}$, we may define a Fredholm series $\operatorname{det}\left(1-X f \mid C_{G, K^{p}, \lambda, \text { cusp }}^{\bullet}\right)$. If $P_{+}(X)=\prod_{i} \operatorname{det}\left(1-X f \mid C_{G, K^{p}, \lambda, \text { cusp }}^{i}\right)$ has a factorization $P_{+}=Q_{+} S_{+}$with $Q_{+}$a polynomial with invertible leading coefficient, then this factorization induces a decomposition $C_{G, K^{p}, \lambda, \text { cusp }}^{\bullet}=N^{\bullet} \oplus F^{\bullet}$.

\section{Theory of Determinants}

In order to construct the pieces of the eigenvariety, we will make use of the theory of determinants. We will recall some basic definitions from Che14 and prove a lemma concerning the ratio of two determinants. 
Definition 6.1. Let $A$ be commutative ring, and let $R$ be an $A$-module. An $A$-valued polynomial law on $R$ is a rule that assigns to any commutative $A$-algebra $B$ a map of sets $D_{B}: R \otimes_{A} B \rightarrow B$ that is functorial in the sense that for any $A$-algebra homomorphism $f: B \rightarrow B^{\prime}$,

$$
D_{B^{\prime}} \circ\left(\operatorname{id}_{R} \otimes f\right)=f \circ D_{B}
$$

Let $d$ be a nonnegative integer. We say that a polynomial law $D$ is homogeneous of degree $d$ if

$$
D_{B}(b r)=b^{d} D_{B}(r) \quad \forall B, b \in B, r \in R \otimes_{A} B
$$

Now assume that $R$ is an $A$-algebra. We say that a polynomial law $D$ is multiplicative if

$$
D_{B}(1)=1, \quad D_{B}\left(r r^{\prime}\right)=D_{B}(r) D_{B}\left(r^{\prime}\right) \quad \forall B, r, r^{\prime} \in R \otimes B .
$$

We say that a polynomial law $D$ is a determinant of dimension $d$ if it is homogeneous of degree $d$ and multiplicative.

Let $M$ be an $R$-module that is projective of rank $d$ as an $A$-module. Then the rule that sends $r \in R \otimes_{A} B$ to $\operatorname{det}\left(r \mid M \otimes_{A} B\right)$ is a determinant of dimension $d$.

Lemma 6.2 ([Rob63, Proposition I.1]). Let $A$ be a commutative ring, and let $R$ be an A-module. Let $D$ be an A-valued polynomial law on $R$ that is homogeneous of degree $d$, let $n$ be a positive integer, and let $r_{1}, \ldots, r_{n} \in R$. Then $D_{A\left[X_{1}, \ldots, X_{n}\right]}\left(X_{1} r_{1}+\ldots+X_{n} r_{n}\right)$ is a homogeneous polynomial of degree $d$ in $X_{1}, \ldots, X_{n}$.

Lemma 6.3. Let $A$ be a commutative ring, let $R$ be an $A$-algebra, and let $D^{+}, D^{-}$ be $A$-valued determinants on $R$ of dimension $d_{+}, d_{-}$, respectively, with $d_{+} \geq d_{-}$. Let $d=d_{+}-d_{-}$. There is at most one determinant $D$ of dimension $d$ satisfying $D_{B}^{+}(r)=D_{B}^{-}(r) D_{B}(r)$ for all $A$-algebras $B$ and all $r \in R \otimes_{A} B$. 
The following are equivalent:

(1) There exists a determinant D satisfying the above condition.

(2) For any commutative A-algebra $B$ and $r \in R \otimes_{A} B$,

$$
D_{B \llbracket X \rrbracket}^{+}(1+X r) / D_{B \llbracket X \rrbracket}^{-}(1+X r)
$$

is a polynomial of degree at most $d$ in $X$.

(3) For any positive integer $n$ and $r_{1}, \ldots, r_{n} \in R$,

$$
D_{A \llbracket X_{1}, \ldots, X_{n} \rrbracket}^{+}\left(1+X_{1} r_{1}+\ldots+X_{n} r_{n}\right) / D_{A \llbracket X_{1}, \ldots, X_{n} \rrbracket}^{-}\left(1+X_{1} r_{1}+\ldots+X_{n} r_{n}\right)
$$

is a polynomial of total degree at most $d$ in $X_{1}, \ldots, X_{n}$.

Proof. If $D_{B}^{-}(r)$ is a unit, then we we will define $F_{B}(r):=D_{B}^{+}(r) / D_{B}^{-}(r)$.

If $D$ is a determinant satisfying the conditions of the lemma, then $F_{B \llbracket X \rrbracket}(1+X r)=$ $D_{B \llbracket X \rrbracket}(1+X r)$ for all $B, r \in R \otimes_{A} B$. Furthermore, $D_{B}(r)$ must be the coefficient of $X^{d}$ in $D_{B \llbracket X \rrbracket}(1+X r)$, since functoriality implies that both of these quantities must equal the coefficient of $X^{d}$ in $D_{B[X, Y]}(Y+X r)$. So $D$ is uniquely determined if it exists.

Lemma 6.2 proves $(1) \Rightarrow(3)$, and $(3) \Rightarrow(2)$ follows from functoriality.

Now we will show that $(2) \Rightarrow(1)$. Assume that condition (2) holds. We define $D_{B}(r)$ be the coefficient of $X^{d}$ in $F_{B \llbracket X \rrbracket}(1+X r)$. We know that $D_{B \llbracket X \rrbracket}^{+}(1+X r)$ (resp. $\left.D_{B \llbracket X \rrbracket}^{-}(1+X r), F_{B \llbracket X \rrbracket}(1+X r)\right)$ is a polynomial of degree at most $d_{+}$(resp. $d_{-}$, $d)$ and the coefficient of $X^{d_{+}}\left(\operatorname{resp} . X^{d_{-}}, X^{d}\right)$ is $D_{B}^{+}(r)\left(\operatorname{resp} . D_{B}^{-}(r), D_{B}(r)\right)$. So $D_{B}^{+}(r)=D_{B}^{-}(r) D_{B}(r)$.

It remains to show that $D$ is a determinant. Since $D^{+}, D^{-}$are functorial, $D$ is as well. To show that $D$ is homogeneous of degree $d$, we observe that the map $X \mapsto b X$ multiplies the coefficient of $X^{d}$ in $F_{B \llbracket X \rrbracket}(1+X r)$ by $b^{d}$. 
Finally, we check that $D$ is multiplicative. We have $F_{B \llbracket X \rrbracket}(1+X)=(1+X)^{d}$, so $D_{B}(1)=1$. Observe that $D\left(r_{1} r_{2}\right)$ is the coefficient of $\left(X_{1} X_{2}\right)^{d}$ in $D_{B\left[X_{1}, X_{2}\right]}(1+$ $\left.X_{1} X_{2} r_{1} r_{2}\right)$, while $D\left(r_{1}\right) D\left(r_{2}\right)$ is the coefficient of $\left(X_{1} X_{2}\right)^{d}$ in $D_{B\left[X_{1}, X_{2}\right]}\left(1+X_{1} r_{1}+\right.$ $\left.X_{2} r_{2}+X_{1} X_{2} r_{1} r_{2}\right)$. The equality of these coefficients follows from Lemma 6.2 applied to $1, r_{1}, r_{2}, r_{1} r_{2}$.

Definition 6.4. Let $D$ be an $A$-valued determinant on $R$. We denote by $\operatorname{ker}(D)$ the set of $r \in R$ such that for all $B$ and all $r^{\prime} \in B \otimes_{A} R, D_{B}\left(1+r^{\prime} r\right)=1$.

\section{Construction of the Eigenvariety}

7.1. Weight space and Fredholm series. Now we return to the setup of sections 445. We continue to assume that $G(\mathbb{R})$ has discrete series. Let $T^{\prime}$ be the quotient of $T_{0}$ by the closure of $Z_{G}(\mathbb{Q}) G_{\infty}^{+} K^{p} \cap T_{0}$. We define the weight space

$$
\mathcal{W}:=\operatorname{Spa}\left(\mathbb{Z}_{p} \llbracket T^{\prime} \rrbracket, \mathbb{Z}_{p} \llbracket T^{\prime} \rrbracket\right)^{\text {an }}
$$

Let $\mathcal{U}=\operatorname{Spa}\left(A, A^{+}\right)$be an open affinoid subset of $\mathcal{W}$ with $A$ a complete Tate ring. Let $\lambda: T_{0} \rightarrow A^{\times}$be the tautological character induced by the map $T_{0} \rightarrow T^{\prime} \rightarrow \mathbb{Z}_{p} \llbracket T^{\prime} \rrbracket$. For any $f \in \mathcal{H}_{G}^{\prime} \otimes_{\mathbb{Z}_{p}} A$, let

$$
P_{f}(X):=\operatorname{det}\left(1-X f \mid C_{G, K^{p}, \lambda, \text { cusp }}^{\bullet}\right)^{(-1)^{d / 2}} .
$$

Note that $d=\operatorname{dim} S_{G}(K)$ is even since $G(\mathbb{R})$ has discrete series. If $\mathcal{V}$ is an open subspace of $\mathcal{W}$, and $f \in \mathcal{H}_{G}^{\prime} \otimes_{\mathbb{Z}_{p}} \mathcal{O}_{\mathcal{W}}(\mathcal{V})$, then we can define $P_{f}(X)$ by gluing.

Definition 7.1.1. Let $\mathcal{V}$ be an open subspace of $\mathcal{W}$. A series $f \in \mathcal{O}_{\mathcal{W}}(\mathcal{V}) \llbracket X \rrbracket$ is called a Fredholm series if it is the power series expansion of some global section of $\mathcal{V} \times \mathbb{A}^{1}$ and its leading coefficient is 1 . 
This definition agrees with Definition 2.2.1 if $\mathcal{V}=\operatorname{Spa}\left(A, A^{+}\right)$with $A$ a complete Tate ring.

Proposition 7.1.2. For $f \in \mathcal{H}_{G}^{\prime}$, the series $P_{f}(X) \in \mathcal{O}_{\mathcal{W}}(\mathcal{W}) \llbracket X \rrbracket$ is a Fredholm series.

Proof. It suffices to check that the restriction of $P_{f}$ to each irreducible component $\mathcal{V}$ of $\mathcal{W}$ is a Fredholm series. The ring $\mathcal{O}_{\mathcal{V}}(\mathcal{V})=\mathcal{O}_{\mathcal{V}}^{+}(\mathcal{V})$ is just the completed group ring of the torsionfree part of $T^{\prime}$. In particular, this ring is adic, and its topology is induced by any norm corresponding to a Gauss point of $\mathcal{V}$. So it suffices to check that the restriction of $P_{f}(X)$ to some Gauss point of $\mathcal{V}$ is a Fredholm series. The Gauss points are characteristic zero points, so we may apply the argument of Urb11, Theorem 4.7.3iii] along with Proposition 5.5.1.

We will write $P(X)$ for $P_{u_{t}}(X)$. We define the spectral variety $\mathcal{Z} \subset \mathcal{W} \times \mathbb{A}^{1}$ to be the zero locus of $P(X)$, and we define $w: \mathcal{Z} \rightarrow \mathcal{W}$ to be the projection. We also define

$$
P_{+}(X):=\prod_{i} \operatorname{det}\left(1-X u_{t} \mid C_{G, K^{p}, \lambda, \text { cusp }}^{i}\right)
$$

7.2. Pieces of the eigenvariety. Now we construct the individual pieces of the eigenvariety. Let $z \in \mathcal{Z}$. By AIP, Corollaire B.1], there exists an open affinoid neighborhood $\mathcal{U}=\operatorname{Spa}\left(A, A^{+}\right)$of $w(z)$ and a factorization $P_{+}(X)=Q_{+}(X) S_{+}(X)$, with $Q_{+}(X) \in A[X], S_{+}(X) \in A\{\{X\}\}$, such that $Q_{+}(X)$ and $S_{+}(X)$ are relatively prime, $Q_{+}$vanishes at $x$, and the leading coefficient of $Q_{+}$is invertible. The factorization of $P_{+}$induces a factorization $P(X)=Q(X) S(X)$ satisfying similar properties. The factorization also determines a sub-complex $N^{\bullet}$ of $C_{G, K^{p}, \lambda, \text { cusp }}^{\bullet}$, as described in sections 4.4 and 5.5 . 
Proposition 7.2.1. Let $D^{+}$be the determinant associated with the action of $\mathcal{H}_{G} \otimes_{\mathbb{Z}_{p}} A$ on $\bigoplus_{i \equiv d / 2(2)} N^{i}$, and let $D^{-}$be the determinant associated with the action of $\mathcal{H}_{G} \otimes_{\mathbb{Z}_{p}} A$ on $\bigoplus_{i \equiv d / 2+1(2)} N^{i}$. Then there exists a determinant $D$ so that $D^{+}=D D^{-}$.

Proof. Let $R=\mathcal{H}_{G} \otimes_{\mathbb{Z}_{p}} A$. As before, if $B$ is an $A$-algebra and $r \in R \otimes_{A} B$ has the property that $D_{B}^{-}(r)$ is invertible, we write $F_{B}(r)$ for the ratio $D_{B}^{+}(r) / D_{B}^{-}(r)$.

First, we will show that for any $r \in R$, the polynomial $D_{A[X]}^{-}(1+X r)$ divides the polynomial $D_{A[X]}^{+}(1+X r)$. It suffices to check that this holds at a Zariski dense subset of points of $\mathcal{U}$. By [Urb11, Lemma 4.1.12 and Theorem 4.7.3iii], it holds at all rigid analytic points. So $D_{A[X]}^{-}(1+X r)$ divides $D_{A[X]}^{+}(1+X r)$, and hence $F_{A \llbracket X \rrbracket}(1+X r)$ must be a polynomial.

For any $z \in \mathbb{Z}_{p}, F_{A \llbracket X \rrbracket}(1+X(r+z))$ is a polynomial. Let $d_{+}$and $d_{-}$be the dimensions of $D^{+}$and $D^{-}$, respectively, and let $d=d_{+}-d_{-}$. For almost all $z$, $D_{A \llbracket X \rrbracket}^{-}(1+X(r+z))$ has degree $d_{-} ;$choose one such $z$. Then $F_{A \llbracket X \rrbracket}(1+X(r+z))$ has degree at most $d$. So $F_{A \llbracket X \rrbracket}(1+X r)=(1-X z)^{d} F_{A \llbracket X \rrbracket}\left(1+X(1-X z)^{-1}(r+z)\right)$ has degree at most $d$ as well.

Next, we observe that, since $F_{A \llbracket X \rrbracket}\left(1+X\left(z_{1} r_{1}+\ldots+z_{n} r_{n}\right)\right)$ is a polynomial of degree at most $d$ for all $z_{1}, \ldots, z_{n} \in \mathbb{Z}_{p}, r_{1}, \ldots, r_{n} \in R, F_{A \llbracket X_{1}, \ldots, X_{n} \rrbracket}\left(1+X_{1} r_{1}+. .+X_{n} r_{n}\right)$ must be a polynomial of total degree at most $d$. Then we may apply Lemma 6.3.

We let $h_{\mathcal{U}, Q_{+}}=\left(\mathcal{H}_{G} \otimes_{\mathbb{Z}_{p}} A\right) / \operatorname{ker}(D)$. Since $\operatorname{ker}(D)$ contains any operator that annihilates $N^{\bullet}, h_{\mathcal{U}, Q_{+}}$must be finitely generated as an $A$-module. We use the extension $h_{\mathcal{U}, Q_{+}} \rightarrow A$ to construct an adic space $\mathcal{E}_{\mathcal{U}, Q_{+}}$over $U$ as follows. We give $h_{\mathcal{U}, Q_{+}}$the "A-module topology" defined in Hub94, Section 2], and we let $h_{U, Q}^{+}$be the normal closure of $A^{+}$in $h_{U, Q}$. We define $\mathcal{E}_{\mathcal{U}, Q_{+}}=\operatorname{Spa}\left(h_{\mathcal{U}, Q_{+}}, h_{\mathcal{U}_{,} Q_{+}}^{+}\right)$. 
Since $Q^{*}(X)$ is the characteristic polynomial of $u$ acting on $N^{\bullet}$, it follows from Che14, Lemma 1.12iv] that $Q^{*}(u)$ is in $\operatorname{ker}(D)$, and so there is a canonical map $\mathcal{E}_{\mathcal{U}, Q_{+}} \rightarrow \mathcal{Z}$

7.3. Gluing. We will glue the $\mathcal{E}_{\mathcal{U}, Q_{+}}$as in [Buz07, Section 5]. We need the following lemma to verify that the pieces can be glued.

Lemma 7.3.1. If $\mathcal{U}^{\prime} \subset \mathcal{U}$, then there is a canonical isomorphism $\mathcal{E}_{\mathcal{U}^{\prime}, Q_{+}} \cong \mathcal{E}_{\mathcal{U}_{,} Q_{+}} \times_{\mathcal{U}}$ $\mathcal{U}^{\prime}$

Proof. The restriction map $\mathcal{O}_{\mathcal{W}}(\mathcal{U}) \rightarrow \mathcal{O}_{\mathcal{W}}\left(\mathcal{U}^{\prime}\right)$ is étale by Hub96, Proposition 1.6.7i and Corollary 1.7.3iii]. By Ryd08, Theorem I.2.3.2], $h_{\mathcal{U}^{\prime}, Q_{+}} \cong h_{\mathcal{U}, Q_{+}} \otimes_{\mathcal{O}_{\mathcal{W}}(\mathcal{U})} \mathcal{O}_{\mathcal{W}}\left(\mathcal{U}^{\prime}\right)$. Since $h_{\mathcal{U}, Q_{+}}$is finite over $\mathcal{O}_{\mathcal{W}}\left(\mathcal{U}^{\prime}\right)$, it follows that $\mathcal{E}_{\mathcal{U}^{\prime}, Q_{+}} \cong \mathcal{E}_{\mathcal{U}, Q_{+}} \times_{\mathcal{U}} \mathcal{U}^{\prime}$.

One can also show, using essentially the same proof as Urb11, Proposition 5.3.5], that if $Q_{+}$and $Q_{+}^{\prime}$ are relatively prime, then there is a canonical isomorphism $\mathcal{E}_{\mathcal{U}, Q_{+} Q_{+}^{\prime}} \cong \mathcal{E}_{\mathcal{U}, Q_{+}} \sqcup \mathcal{E}_{\mathcal{U}, Q_{+}^{\prime}}$

Theorem 7.3.2. The $\mathcal{E}_{\mathcal{U}_{, Q_{+}}}$can be glued to form an adic space $\mathcal{E}$. Furthermore, $\mathcal{E}$ is equidimensional in the sense of [Hub96, Definition 1.8.1] and the morphism $\mathcal{E} \rightarrow \mathcal{Z}$ is finite and surjective.

Proof. To show that the morphism $\mathcal{E} \rightarrow \mathcal{Z}$ is finite, we observe that $\mathcal{Z}$ can be covered by open sets whose preimage in $\mathcal{E}$ is contained in some $\mathcal{E}_{\mathcal{U}, Q_{+}}$. The finiteness of the morphism $\mathcal{E} \rightarrow \mathcal{Z}$ then follows from the finiteness of the maps $\mathcal{E}_{\mathcal{U}_{,} Q_{+}} \rightarrow \mathcal{U}$

Now we check that the morphism is surjective. Let $z \in \mathcal{Z}$, and let $k$ be the residue field of $z$. Observe that $\operatorname{Spec} \mathcal{H}_{G} \rightarrow \operatorname{Spec} \mathbb{Z}_{p}\left[u_{t}\right]$ is surjective, so $\mathcal{H}_{G} \otimes_{\mathbb{Z}_{p}\left[u_{t}\right]} k$ cannot be the zero ring. The image of $\operatorname{ker}(D)$ in $\mathcal{H}_{G} \otimes_{\mathbb{Z}_{p}\left[u_{t}\right]} k$ is contained in the kernel of the base change of $D$ to $\mathcal{H}_{G} \otimes_{\mathbb{Z}_{p}\left[u_{t}\right]} k$. Therefore the image of $\operatorname{ker}(D)$ cannot be the unit ideal, and so there must be a point of $\mathcal{E}$ lying above $z$. 
Finally, we show that $\mathcal{E}$ is equidimensional. The characteristic zero locus of $\mathcal{W}$ can be covered by open affinoids $\mathcal{U}=\operatorname{Spa}\left(A, A^{+}\right)$with $A$ an integral domain. Then for any $Q, h_{\mathcal{U}, Q}$ will be a torsionfree $A$-module, so the portion of $\mathcal{E}$ lying over $\mathcal{U}$ will be equidimensional. For any affinoid $\mathcal{U}=\operatorname{Spa}\left(A, A^{+}\right) \subset X, p$ is not a zero divisor in $A$, so $\mathcal{E}$ cannot have any components that consist solely of characteristic $p$ points.

\section{REFERENCES}

[AIP] F. Andreatta, A. Iovita, and V. Pilloni. Le halo spectral. Ann. Sci. École Norm. Sup. To appear.

[Ami64] Y. Amice. Interpolation p-adique. Bull. Soc. Math. France, 92:117-180, 1964.

[AS08] A. Ash and G. Stevens. p-adic deformations of arithmetic cohomology. 2008.

[BK05] K. Buzzard and L. J. P. Kilford. The 2-adic eigencurve at the boundary of weight space. Compos. Math., 141(3):605-619, 2005.

[Buz07] K. Buzzard. Eigenvarieties. In L-functions and Galois representations, volume 320 of London Math. Soc. Lecture Note Ser., pages 59-120. Cambridge Univ. Press, Cambridge, 2007.

[BW00] A. Borel and N. Wallach. Continuous cohomology, discrete subgroups, and representations of reductive groups, volume 67 of Mathematical Surveys and Monographs. American Mathematical Society, Providence, RI, second edition, 2000.

[BZ77] I. N. Bernstein and A. V. Zelevinsky. Induced representations of reductive $p$-adic groups. I. Ann. Sci. École Norm. Sup. (4), 10(4):441-472, 1977.

[Che14] G. Chenevier. The $p$-adic analytic space of pseudocharacters of a profinite group and pseudorepresentations over arbitrary rings. In Automorphic forms and Galois representations. Vol. 1, volume 414 of London Math. Soc. Lecture Note Ser., pages 221-285. Cambridge Univ. Press, Cambridge, 2014.

[CM98] R. Coleman and B. Mazur. The eigencurve. In Galois representations in arithmetic algebraic geometry (Durham, 1996), volume 254 of London Math. Soc. Lecture Note Ser., pages 1-113. Cambridge Univ. Press, Cambridge, 1998.

[Col96] R. F. Coleman. Classical and overconvergent modular forms. Invent. Math., 124(1-3):215$241,1996$.

[Col97] R. F. Coleman. p-adic Banach spaces and families of modular forms. Invent. Math., 127(3):417-479, 1997.

[Col10] P. Colmez. Fonctions d'une variable p-adique. Astérisque, 330:13-59, 2010. 
[Eme06a] M. Emerton. Jacquet modules of locally analytic representations of $p$-adic reductive groups. I. Construction and first properties. Ann. Sci. École Norm. Sup. (4), 39(5):775839, 2006.

[Eme06b] M. Emerton. On the interpolation of systems of eigenvalues attached to automorphic Hecke eigenforms. Invent. Math., 164(1):1-84, 2006.

[Han15] D. Hansen. Universal eigenvarieties, trianguline Galois representations, and p-adic Langlands functoriality. J. Reine Angew. Math., 2015.

[Hid86] H. Hida. Galois representations into $\mathrm{GL}_{2}\left(\mathbf{Z}_{p}[[X]]\right)$ attached to ordinary cusp forms. Invent. Math., 85(3):545-613, 1986.

[Hid88] H. Hida. On p-adic Hecke algebras for $\mathrm{GL}_{2}$ over totally real fields. Ann. of Math. (2), 128(2):295-384, 1988.

[Hid94] H. Hida. p-adic ordinary Hecke algebras for GL(2). Ann. Inst. Fourier (Grenoble), 44(5):1289-1322, 1994.

[Hub94] R. Huber. A generalization of formal schemes and rigid analytic varieties. Math. Z., 217(4):513-551, 1994.

[Hub96] R. Huber. Étale cohomology of rigid analytic varieties and adic spaces. Aspects of Mathematics, E30. Friedr. Vieweg \& Sohn, Braunschweig, 1996.

[JN] C. Johansson and J. Newton. Extended eigenvarieties for overconvergent cohomology. arXiv:1604.07739.

[Kat73] N. M. Katz. p-adic properties of modular schemes and modular forms. In Modular functions of one variable, III (Proc. Internat. Summer School, Univ. Antwerp, Antwerp, 1972), pages 69-190. Lecture Notes in Mathematics, Vol. 350. Springer, Berlin, 1973.

[Laz65] M. Lazard. Groupes analytiques p-adiques. Inst. Hautes Études Sci. Publ. Math., 26:389$603,1965$.

[LS04] J.-S. Li and J. Schwermer. On the Eisenstein cohomology of arithmetic groups. Duke Math. J., 123(1):141-169, 2004.

[LWX17] R. Liu, D. Wan, and L. Xiao. The eigencurve over the boundary of weight space. Duke Math. J., 166(9):1739-1787, 2017.

[Rob63] N. Roby. Lois polynomes et lois formelles en théorie des modules. Ann. Sci. École Norm. Sup. (3), 80:213-348, 1963.

[Ryd08] D. Rydh. Families of cycles and the Chow scheme. PhD thesis, KTH, 2008.

[Ste94] G. Stevens. Rigid analytic modular symbols. 1994.

[Urb11] E. Urban. Eigenvarieties for reductive groups. Ann. of Math. (2), 174(3):1685-1784, 2011. 